COMMUNICATIONS IN

ANALYSIS AND GEOMETRY

Volume 14, Number 5, 847-881, 2006

\title{
Harmonic morphisms between Weyl spaces and twistorial maps
}

\author{
Eric Loubeau and Radu Pantilie
}

\begin{abstract}
We show that Weyl spaces provide a natural context for harmonic morphisms, and we give the necessary and sufficient conditions under which on an Einstein-Weyl space of dimension 4 there can be defined, locally, at least five distinct foliations of dimension 2 which produce harmonic morphisms (Theorem 7.4). Also, we describe the harmonic morphisms between Einstein-Weyl spaces of dimensions 4 and 3 (Theorem 7.6).
\end{abstract}

\section{Introduction}

Harmonic morphisms between Riemannian manifolds are smooth maps which pull back (local) harmonic functions to harmonic functions. By the basic characterization theorem of Fuglede and Ishihara [8,14], harmonic morphisms are harmonic maps which are horizontally weakly conformal. See [11] for a frequently updated list of publications on harmonic morphisms.

The simplest nontrivial examples of harmonic morphisms are given by harmonic functions from a two-dimensional oriented conformal manifold: any such harmonic morphism is, locally, the sum of a $(+)$ holomorphic function and a (-)holomorphic function (see [3]). Similar descriptions, in higher dimensions, can be obtained if instead of $( \pm$ )holomorphic functions, we use the more general notion of twistorial map [26]. A twistorial structure on a complex manifold $M$ is given by a foliation $\mathscr{F}$ on a complex manifold $P$ such that $\mathscr{F} \cap \operatorname{ker} d \pi=\{0\}$, where $\pi: P \rightarrow M$ is a proper complex analytic submersion; the leaf space of $\mathscr{F}$ is called the twistor space of the given twistorial structure $(P, M, \pi, \mathscr{F})$ and is usually denoted $Z(M)$. It follows that, locally, we can find sections of $\pi$ whose images are foliated by leaves of $\mathscr{F}$; by projecting back through $\pi$, we endow $M$ with a sheaf of complex analytic submersions. Locally, a twistorial function is the composition of such a submersion followed by a complex analytic function.

A sufficient condition for a complex analytic map $\varphi: M \rightarrow N$ between complex manifolds endowed with twistorial structures $\left(P, M, \pi_{P}, \mathscr{F}\right)$ and $\left(Q, N, \pi_{Q}, \mathscr{G}\right)$ to be twistorial, with respect to a map $\Phi: P \rightarrow Q$, is 
$\pi_{Q} \circ \Phi=\varphi \circ \pi_{P}$ and $d \Phi(\mathscr{F})=\mathscr{G}$; then, $\Phi$ induces a map between the twistor spaces of $\left(P, M, \pi_{P}, \mathscr{F}\right)$ and $\left(Q, N, \pi_{Q}, \mathscr{G}\right)$ which we call the twistorial representation of $\varphi$ (with respect to $\Phi$ ). To define the notion of twistorial map, it is not necessary to assume that $\mathscr{F}$ and $\mathscr{G}$ are integrable; that is, we can speak about twistorial maps between manifolds endowed with almost twistorial structures (see $[26, \S 3]$ for the definition of twistorial map in the category of complex manifolds and [20] for the definitions of almost twistorial structure and twistorial map in the smooth category).

Thus, any twistorial structure on $M$ determines a sheaf of twistorial functions $\mathcal{F}_{M}$ on $M$; such sheaves can be obtained by complexifying the following examples of complex valued functions:

(i) ( \pm Holomorphic functions on a Hermitian manifold; here $(M, c, J)$ is the (germ-unique) complexification of a Hermitian manifold, $P=$ $M_{+} \sqcup M_{-}$, with $M_{ \pm}=M$, and the twistor distribution $\left.\mathscr{F}\right|_{M_{ \pm}}$is the (干i)eigendistribution of $J$ (cf. [26, Example 2.3]).

(ii) Functions on a three-dimensional Einstein-Weyl space, which, locally, are the composition of a horizontally conformal submersion with geodesic fibres followed by a complex analytic function; here $\left(M^{3}, c, D\right)$ is the complexification of a three-dimensional Einstein-Weyl space, $\pi: P \rightarrow M$ is the bundle of two-dimensional degenerate spaces on $\left(M^{3}, c\right)$ and, for each $p \in P$, the subspace $\mathscr{F}_{p} \subseteq T_{p} P$ is the horizontal lift of $p \subseteq T_{\pi(p)} M$ with respect to $D$ (see [26, Example 2.4] for details about this twistorial structure).

(iii) Functions on a four-dimensional anti-self-dual manifold, which are holomorphic with respect to a (local) positive Hermitian structure on it; here $\left(M^{4}, c\right)$ is the complexification of a four-dimensional antiself-dual manifold, $\pi: P \rightarrow M$ is the bundle of self-dual spaces on $\left(M^{4}, c\right)$ and, for each $p \in P$, the subspace $\mathscr{F}_{p} \subseteq T_{p} P$ is the horizontal lift of $p \subseteq T_{\pi(p)} M$ with respect to the Levi-Civita connection of any local representative of $c$ (see [26, Example 2.6] for details about this twistorial structure).

One of the main steps in the process of classifying harmonic morphisms is to prove that these are twistorial. For example, any harmonic morphism from an Einstein manifold of dimension 4 with fibres of dimension 1 or 2 is twistorial ([23, 26, 29]; cf. Corollaries 7.1 and 7.2 below).

This paper gives an answer to the following question of John C. Wood: Can (the submersive) twistorial maps be seen as harmonic morphisms? If we restrict ourselves to twistorial maps which pull back twistorial functions to 
twistorial functions (for example, if we work with twistorial maps, as above, with the further property that their twistorial representation is submersive), then the answer, in the affirmative, to this question follows if we work with sheaves of twistorial functions $\mathcal{F}_{M}$ for which there exists a sheaf of "harmonic" functions $\mathcal{L}$ such that $\mathcal{F}_{M} \cap \mathcal{L}$ is a "sufficiently large" subsheaf of $\mathcal{L}$ (in particular, if the sheaf of vector spaces generated by $\mathcal{F}_{M} \cap \mathcal{L}$ is equal to $\mathcal{L}$, like in the case of two-dimensional conformal manifolds). We argue that for each of the examples (i), (ii) and (iii), above, a good candidate for $\mathcal{L}$ can be obtained by endowing the given conformal structure with a suitable Weyl connection (the obvious one for (ii)).

The definition of harmonic functions on a Weyl space is given in Section 2; there we also show that the basic theorem of Fuglede and Ishihara generalizes to harmonic morphisms between Weyl spaces. In Section 3, we do the same for the fundamental equation for harmonic morphisms (see [3]).

In Section 4, we recall the definition and the basic properties of the Weyl connection of an almost Hermitian manifold [28]; we show that for Hermitian manifolds, this is characterized by the property that all the $( \pm)$ holomorphic functions are harmonic with respect to it. We also prove that any holomorphic horizontally weakly conformal map between almost Hermitian manifolds endowed with their Weyl connections is harmonic and, hence, a harmonic morphism (cf. [12,18]).

In Section 5, we recall from [26] the basic examples of twistorial maps and study the conditions under which these are harmonic morphisms; the resulting harmonic morphisms are between Weyl spaces of dimensions $m$ and $n$ where $(m, n)=(3,2),(4,2),(4,3)$. Thus, we obtain the following for an analytic submersion $\varphi$ between Weyl spaces of dimensions $m$ and $n$.

- If $(m, n)=(3,2)$, then $\varphi$ is twistorial if and only if it is a harmonic morphism (Example 5.1).

- If $(m, n)=(4,2),(4,3)$, then we obtain necessary and sufficient conditions under which $\varphi$ is a twistorial harmonic morphism (Example 5.2, Theorem 5.4).

The main purpose of Section 6 is to prove that if $\varphi$ is a harmonic morphism from a Weyl space of dimension 4 to a Weyl space of dimension 2 or 3 , then $\varphi$ is twistorial if and only if the difference between the Ricci tensor of the domain and the pull-back of the Ricci tensor of the codomain is zero along the horizontal null directions (Propositions 6.3 and 6.6).

In Section 7, we apply the results of Sections 5 and 6 to study harmonic morphisms from four-dimensional Einstein-Weyl spaces. Thus, 
- we give the necessary and sufficient conditions under which on an Einstein-Weyl space of dimension 4 there can be defined, locally, at least five distinct foliations of dimension 2 which produce harmonic morphisms (Theorem 7.4);

- we describe the harmonic morphisms between Einstein-Weyl spaces of dimensions 4 and 3 (Theorem 7.6).

We are grateful to Paul Baird for his interest in this work and to John C. Wood for useful comments.

\section{Harmonic morphisms between Weyl spaces}

In this section, we shall work in the smooth and (real or complex) analytic categories; unless otherwise stated, all the manifolds are assumed to be connected. A conformal manifold $\left(M^{m}, c\right)$ is a manifold endowed with a reduction of its frame bundle to $C O(m, \mathbb{K}),(\mathbb{K}=\mathbb{R}, \mathbb{C})$. We shall denote by $L^{2}$ the line bundle associated to the bundle of conformal frames of $\left(M^{m}, c\right)$ via the morphism of Lie groups $\rho_{m}: C O(m, \mathbb{K}) \rightarrow \mathbb{K} \backslash\{0\}$ characterized by $a^{\mathrm{T}} a=\rho_{m}(a) I_{m}, \quad(a \in C O(m, \mathbb{K}), \mathbb{K}=\mathbb{R}, \mathbb{C})$. In the smooth and real analytic categories, $L^{2}$ is canonically oriented and so it admits a canonical square root, denoted by $L$; moreover, $L$ does not depend on $c$ (see [5]). In the complex analytic category, such a square root can be found locally; furthermore, if $m$ is odd, then there exists a canonical choice for $L$ (this follows from the fact that if $m$ is odd, then there exists a natural isomorphism of Lie groups $\left.C O(m, \mathbb{K})=\mathbb{K}^{*} \times S O(m, \mathbb{K}),(\mathbb{K}=\mathbb{R}, \mathbb{C})\right)$. In the smooth and real analytic categories, positively oriented local sections of $L^{2}$ correspond to local representatives of $c$. In the complex analytic category, nowhere zero local sections of $L^{2}$ correspond to local representatives of $c$. Note that if $b$ is a section of $\otimes^{2} T^{*} M$, then its traces with respect to local representatives of $c$ define a section of $L^{* 2}$ which will be denoted trace ${ }_{c} b$. More generally, if $b$ is a section of $E \otimes\left(\otimes^{2} T^{*} M\right)$ for some vector bundle $E$ over $M$, then we can

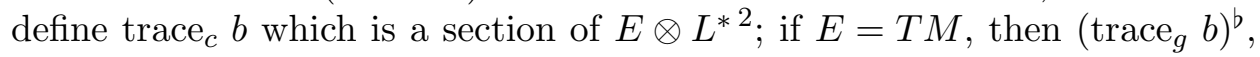
where $g$ is any local representative of $c$, defines a 1-form on $M$ which will be denoted $\left(\operatorname{trace}_{c} b\right)^{b}$ (see $\left.[5,9]\right)$.

The following definition is a simple generalization of the well-known notion of harmonic map (see [3]).

Definition 2.1. Let $(M, c)$ be a conformal manifold, $N$ a manifold and $D^{M}, D^{N}$ linear connections on $M, N$, respectively. 
A map $\varphi:\left(M, c, D^{M}\right) \rightarrow\left(N, D^{N}\right)$ is called harmonic (with respect to $c$, $\left.D^{M}, D^{N}\right)$ if

$$
\operatorname{trace}_{c}(D d \varphi)=0
$$

where $D$ is the connection on $\varphi^{*}(T N) \otimes T^{*} M$ induced by $D^{M}, D^{N}$ and $\varphi$.

There is no loss of generality if we assume $D^{M}$ and $D^{N}$ to be torsionfree. Indeed, Equation (2.1) is unchanged if we replace $D^{M}$ and $D^{N}$ with the associated torsion-free affinely equivalent connections (see [16, Chapter III, Proposition 7.9]).

A harmonic map $\varphi:(M, g) \rightarrow(N, h)$ between Riemannian manifolds is harmonic in the sense of Definition 2.1 if $M$ and $N$ are endowed with the Levi-Civita connections of $g$ and $h$, respectively, and $M$ is considered with the conformal structure determined by $g$.

We shall always consider $\mathbb{K}(=\mathbb{R}, \mathbb{C})$ to be endowed with its conformal structure and canonical connection (here $\mathbb{C}$ is considered to be a onedimensional complex manifold). Clearly, a curve on $(M, D)$ is harmonic if and only if it is a geodesic of $D$.

Let $(M, c)$ be a conformal manifold. A torsion-free conformal connection on $(M, c)$ is called a Weyl connection; if $D$ is a Weyl connection on $(M, c)$, then $(M, c, D)$ is called a Weyl space (see [9]). A function (locally) defined on a Weyl space $(M, c, D)$ will be called harmonic if it is harmonic with respect to $c, D$. If $\operatorname{dim} M=2$, then a function $f$ on the Weyl space $(M, c, D)$ is harmonic if and only if it is harmonic with respect to any local representative of $c$.

Proposition 2.2. Let $\left(M, c_{M}\right)$ be a conformal manifold of dimension $m \neq$ 2 , endowed with a linear connection $D$.

Then there exists a unique Weyl connection $D_{1}$ on $\left(M, c_{M}\right)$ such that

$$
\operatorname{trace}_{c_{M}}(D d f)=\operatorname{trace}_{c_{M}}\left(D_{1} d f\right)
$$

for any function $f$ (locally) defined on $M$.

Proof. For each local representative $g$ of $c_{M}$, we define a (local) 1-form $\alpha^{g}$ by

$$
\alpha^{g}(X)=\frac{1}{m-2} g\left(\operatorname{trace}_{g}\left(\nabla^{g}-D\right), X\right)
$$

for all $X \in T M$, where $\nabla^{g}$ is the Levi-Civita connection of $g$. It is easy to prove that $\alpha^{g \lambda^{-2}}=\alpha^{g}+\lambda^{-1} d \lambda$. Hence, the family of 1-forms $\left\{\alpha^{g}\right\}$ defines a 
connection on $L$. But any connection on $L$ corresponds to a Weyl connection $D_{1}$ on $\left(M, c_{M}\right)$ (see [9], the 1 -form $\alpha^{g}$ is the Lee form of $D_{1}$ with respect to $g$ ). Now, (2.2) is equivalent to (2.3) and the proof follows.

The following definition (cf. $[3,8,14]$ ) will be central in this paper.

Definition 2.3. Let $\left(M, c_{M}, D^{M}\right)$ and $\left(N, c_{N}, D^{N}\right)$ be Weyl spaces.

A map $\varphi:\left(M, c_{M}, D^{M}\right) \rightarrow\left(N, c_{N}, D^{N}\right)$ is called a harmonic morphism if for any harmonic function $f$ defined on some open set $U$ of $N$, such that $\varphi^{-1}(U) \neq \emptyset$, the function $\left.f \circ \varphi\right|_{\varphi^{-1}(U)}$ is harmonic.

Remark 2.4. Proposition 2.2 shows that if $\operatorname{dim} M, \operatorname{dim} N \neq 2$, then Definition 2.3 does not become more general by using linear connections instead of Weyl connections.

Any harmonic morphism between Riemannian manifolds $\varphi:(M, g) \rightarrow$ $(N, h)$ is also a harmonic morphism between Weyl spaces $\varphi:\left(M,[g], \nabla^{g}\right) \rightarrow$ $\left(N,[h], \nabla^{h}\right)$, where $[g],[h]$ are the conformal structures determined by $g, h$ and $\nabla^{g}, \nabla^{h}$ are the Levi-Civita connections of $g, h$, respectively. However, not all harmonic morphisms between Weyl spaces arise in this way (see, for example, Remark 3.3(2), Corollary 4.6 and Example 7.7(2) below).

Definition 2.5 [3]. Let $\left(M, c_{M}, D^{M}\right)$ be a Weyl space. A foliation $\mathscr{V}$ on $M$ produces harmonic morphisms on $\left(M, c_{M}, D^{M}\right)$ if, locally, it can be defined by submersive harmonic morphisms; that is, for any point of $x \in M$, there exists a submersive harmonic morphism $\varphi:\left(U,\left.c_{M}\right|_{U},\left.D^{M}\right|_{U}\right) \rightarrow\left(N, c_{N}, D^{N}\right)$ such that $\left.\mathscr{V}\right|_{U}=\operatorname{ker} d \varphi$, where $U$ is an open neighbourhood of $x$.

Next, we shall prove the Fuglede-Ishihara theorem $([8,14]$, see $[3])$ for harmonic morphisms between Weyl spaces. For this, we apply the standard strategy (see [3]). First, we need an existence result for harmonic functions from Weyl spaces.

Lemma 2.6 (cf. [3]). Let $(M, c, D)$ be a Weyl space and let $x \in M$. Then for any $v \in T_{x}^{*} M$ and any trace-free symmetric bilinear form $b$ on $\left(T_{x} M, c_{x}\right)$, there exists a harmonic function $f$ defined on some open neighbourhood of $x$ such that $d f_{x}=v$ and $(D d f)_{x}=b$.

Proof. This is essentially the same as for harmonic functions on Riemannian manifolds (see [3] and the references therein). 
We shall give a straightforward proof assuming $(M, c, D)$ (real or complex) analytic (cf. [19, Lemma 2], where we assume that the metric is analytic). Let $U$ be the domain of a normal coordinate system $x^{1}, \ldots, x^{m}$ for $D$, centred at $x$, where $m=\operatorname{dim} M$. We may assume $g\left(d x^{m}, d x^{m}\right)=1$, at $x$, for some local representative $g$ of $c$ over $U$. Hence, by passing to a smaller open neighbourhood of $x$, if necessary, we may assume that the hypersurface $S=\left\{x^{m}=0\right\}$ is nowhere degenerate; equivalently, $S$ is noncharacteristic for the second-order differential operator $f \mapsto \operatorname{trace}_{g}(D d f)$.

Let $p=b_{i j} x^{i} x^{j}+v_{i} x^{i}$. Then, by further restricting $U$, if necessary, and by applying the Cauchy-Kovalevskaya theorem, we can find a harmonic function $f$, with respect to $c, D$, defined on $U$ such that $f$ and $p$ are equal up to the first derivatives along $S$; in particular, $d f_{x}=v$. Hence, possibly excepting $\frac{\partial^{2} f}{\left(\partial x^{m}\right)^{2}}(x)$, all the second-order partial derivatives of $f$, at $x$, are equal to the corresponding derivatives of $p$, at $x$. As $f$ is harmonic, $b$ is trace-free with respect to $g$, and $x$ is the centre of the normal system of coordinates $x^{1}, \ldots, x^{m}$, for $D$, the derivatives $\frac{\partial^{2} f}{\left(\partial x^{m}\right)^{2}}(x)$ and $\frac{\partial^{2} p}{\left(\partial x^{m}\right)^{2}}(x)$ are determined by the other second-order partial derivatives, at $x$, of $f$ and $p$, respectively, and hence must be equal. Thus, $(D d f)_{x}=b$.

Remark 2.7. Let $f$ be a harmonic function (locally defined) on a Weyl space $(M, c, D)$ and let $x \in M$ such that $d f_{x} \neq 0$. Then there exists a local representative $g$ of $c$ defined on some neighbourhood $U$ of $x$ such that $f$ is harmonic with respect to $g$ (this follows, for example, from (2.3) applied to $D$ ). However, the sheaf of harmonic functions on $U$ with respect to $c$, $D$, is equal to the sheaf of harmonic functions of $g$ if and only if $D$ is the Levi-Civita connection of $g$.

Let $\left(M, c_{M}\right)$ be a conformal manifold and let $L_{M}$ be the associated line bundle. Note that the conformal structure $c_{M}$ corresponds to a "musical" isomorphism $T^{*} M \stackrel{\sim}{\longrightarrow} T M \otimes L_{M}^{*}$. Hence, the differential of any map $\varphi$ : $\left(M, c_{M}\right) \rightarrow\left(N, c_{N}\right)$ between conformal manifolds has an adjoint $(d \varphi)^{\mathrm{T}}$ which is a section of the vector bundle $\operatorname{Hom}\left(\varphi^{*}(T N), T M\right) \otimes \operatorname{Hom}\left(L_{M}^{2}, \varphi^{*}\left(L_{N}^{2}\right)\right)$.

Definition 2.8 (cf. [3,22]). A map $\varphi:\left(M, c_{M}\right) \rightarrow\left(N, c_{N}\right)$ between conformal manifolds is called horizontally weakly conformal if there exists a section $\Lambda$ of $\operatorname{Hom}\left(L_{M}^{2}, \varphi^{*}\left(L_{N}^{2}\right)\right)$ such that

$$
\left((d \varphi)^{\mathrm{T}}\right)^{*}\left(c_{M}\right)=\Lambda \otimes c_{\varphi^{*}(T N)},
$$


where $c_{\varphi^{*}(T N)}$ is the conformal structure on $\varphi^{*}(T N)$ induced by $\varphi$ and $c_{N} ; \Lambda$ is called the square dilation of $\varphi$. If $\Lambda=\lambda^{2}$ for some section $\lambda$ of $\operatorname{Hom}\left(L_{M}, \varphi^{*}\left(L_{N}\right)\right)$, then $\lambda$ is a dilation of $\varphi$.

Let $\left(M, c_{M}\right)$ be a conformal manifold. A conformal foliation on $\left(M, c_{M}\right)$ is a foliation which is locally defined by horizontally conformal submersions.

In the complex analytic category, let $\varphi:\left(M, c_{M}\right) \rightarrow\left(N, c_{N}\right)$ be a horizontally conformal submersion with nowhere degenerate fibres. Then, each point of $M$ has an open neighbourhood $U$ such that $\left.\varphi\right|_{U}$ admits a dilation. In fact, by passing, if necessary, to a conformal $\mathbb{Z}_{2}$-covering space of $M$, we can suppose that $\varphi$ admits a dilation.

In the smooth (real analytic) category, any horizontally conformal submersion admits a dilation. Furthermore, if $\varphi:\left(M, c_{M}\right) \rightarrow\left(N, c_{N}\right)$ is horizontally weakly conformal, then there exists a continuous section $\lambda$ of $\operatorname{Hom}\left(L_{M}, \varphi^{*}\left(L_{N}\right)\right)$ such that $\Lambda=\lambda^{2}$; moreover, $\lambda$ is smooth (real analytic) over the set of regular points of $\varphi$ (cf. [3]).

Now we can prove the Fuglede-Ishihara theorem for harmonic morphisms between Weyl spaces.

Theorem 2.9 (cf. $[8,14])$. Let $\left(M, c_{M}, D^{M}\right)$ and $\left(N, c_{N}, D^{N}\right)$ be Weyl spaces.

$A \operatorname{map} \varphi:\left(M, c_{M}, D^{M}\right) \rightarrow\left(N, c_{N}, D^{N}\right)$ is a harmonic morphism if and only if it is harmonic with respect to $c_{M}, D^{M}, D^{N}$ and horizontally weakly conformal.

Proof. For any function $f$, locally defined on $M$, a straightforward calculation gives

$$
\operatorname{trace}_{c_{M}}(D d(f \circ \varphi))=d f\left(\operatorname{trace}_{c_{M}}(D d \varphi)\right)+c_{N}\left(D d f,\left((d \varphi)^{\mathrm{T}}\right)^{*}\left(c_{M}\right)\right)
$$

By applying Lemma 2.6 with $b=0$ and for all $v \in T_{x}^{*} N,(x \in N)$, from Equation (2.4), we obtain that $\varphi$ is harmonic with respect to $c_{M}, D^{M}, D^{N}$. Then, by applying again Lemma 2.6 from Equation (2.4), we obtain that for all trace-free symmetric $b \in \otimes^{2} T^{*} N$, we have $c_{N}\left(b,\left((d \varphi)^{\mathrm{T}}\right)^{*}\left(c_{M}\right)\right)=0$. The proof follows.

An immediate consequence of Theorem 2.9 is that any foliation which produces harmonic morphisms is a conformal foliation (cf. [3]). 


\section{The fundamental equation}

In this section we shall work in the smooth and (real or complex) analytic categories. Let $\left(M^{m}, c_{M}\right)$ be a conformal manifold and let $L$ be the associated line bundle on $M$. Let $\mathscr{V} \subseteq T M$ be a nondegenerate distribution, and let $\mathscr{H}=\mathscr{V}^{\perp}$ be its orthogonal complement. Then, $c_{M}$ induces conformal structures $\left.c_{M}\right|_{\mathscr{V}}$ and $\left.c_{M}\right|_{\mathscr{H}}$ on $\mathscr{V}$ and $\mathscr{H}$, respectively. Let $L_{\mathscr{V}}$ and $L_{\mathscr{H}}$ be the line bundles on $M$ determined by the conformal structures $\left.c_{M}\right|_{\mathscr{V}}$ and $c_{M} \mid \mathscr{H}$, respectively. As any local representative of $c_{M}$ induces local representatives of the conformal structures induced on $\mathscr{V}$ and $\mathscr{H}$, we have isomorphisms between $L^{2}, L_{\mathscr{V}}^{2}$ and $L_{\mathscr{H}}^{2}$ (seen as bundles with group $((0, \infty), \cdot)$ in the smooth and real analytic categories); we shall always identify $L^{2}=$ $L_{\mathscr{V}}^{2}=L_{\mathscr{H}}^{2}$ in this way. Conversely, conformal structures on the complementary distributions $\mathscr{V}$ and $\mathscr{H}$ together with an isomorphism between $L_{\mathscr{V}}^{2}$ and $L_{\mathscr{H}}^{2}$ determine a conformal structure on $M$ such that $\mathscr{H}=\mathscr{V} \perp$ [5]. In other words, nondegenerate distributions $\mathscr{V}$ of dimension $m-n$ on $\left(M^{m}, c_{M}\right)$ correspond to reductions of $c_{M}$ to the subgroup $G=\{(a, b) \in C O(m-n) \times$ $\left.C O(n) \mid \rho_{m-n}(a)=\rho_{n}(b)\right\}$ of $C O(m)$. Then, as the morphisms of Lie groups $p_{1}: G \rightarrow C O(m-n),(a, b) \mapsto a$, and $p_{2}: G \rightarrow C O(n),(a, b) \mapsto b$, satisfy $\left.\rho_{m}\right|_{G}=\rho_{m-n} \circ p_{1}=\rho_{n} \circ p_{2}$, we obtain that $\left.c_{M}\right|_{\mathscr{V}}=p_{1}\left(c_{M}\right)$ and $\left.c_{M}\right|_{\mathscr{H}}=$ $p_{2}\left(c_{M}\right)$ are such that $L_{\mathscr{V}}^{2}$ and $L_{\mathscr{H}}^{2}$ are isomorphic to $L^{2}$. Conversely, if $c_{\mathscr{V}}$ and $c_{\mathscr{H}}$ are (the bundles of conformal frames of) conformal structures on the complementary distributions $\mathscr{V}$ and $\mathscr{H}$, respectively, then $c_{\mathscr{V}}+c_{\mathscr{H}}$ is a reduction of the bundle of linear frames on $M^{m}$ to $C O(m-n) \times C O(n)$, and it is easy to see that isomorphisms between $L_{\mathscr{V}}^{2}$ and $L_{\mathscr{H}}^{2}$ correspond to reductions of $L_{\mathscr{V}}^{2} \oplus L_{\mathscr{H}}^{2}$ to $\iota: H \hookrightarrow H \times H, a \mapsto(a, a)$, where $H=((0, \infty), \cdot)$ in the smooth and real analytic categories and $H=(\mathbb{C} \backslash\{0\}, \cdot)$ in the complex analytic category. As $G=\left(\rho_{m-n} \times \rho_{n}\right)^{-1}(\iota(H))$, it follows that reductions of $c_{\mathscr{V}}+c_{\mathscr{H}}$ to $G$ correspond to isomorphisms between $L_{\mathscr{V}}^{2}$ and $L_{\mathscr{H}}^{2}$; any such reduction determines a conformal structure $c_{M}$ on $M^{m}$ such that $\mathscr{H}=\mathscr{V} \perp$ and $\left.c_{M}\right|_{\mathscr{V}}=c_{\mathscr{V}},\left.c_{M}\right|_{\mathscr{H}}=c_{\mathscr{H}}$.

Example 3.1 [4]. Let $M$ be a manifold endowed with two complementary distributions $\mathscr{V}$ and $\mathscr{H}$. The Bott partial connection $D^{\text {Bott }}$ on $\mathscr{V}$, over $\mathscr{H}$, is defined by $D_{X}^{\mathrm{Bott}} U=\mathscr{V}[X, U]$ for local sections $X$ of $\mathscr{H}$ and $U$ of $\mathscr{V}$.

Suppose that $M$ is endowed with a conformal structure $c_{M}$ with respect to which $\mathscr{V}$ is nondegenerate and $\mathscr{H}=\mathscr{V}^{\perp}$. As $\left(L^{2}\right)^{m-n}=\left(\Lambda^{m-n \mathscr{V}}\right)^{2}$, where $m=\operatorname{dim} M$ and $n$ is the dimension of the distribution $\mathscr{H}$, we have that $D^{\text {Bott }}$ induces a partial connection on $L$ which will also be denoted $D^{\text {Bott }}$; the local connection form of this connection with respect to a local 
section of $L$, corresponding to a local representative $g$ of $c_{M}$, is $\frac{1}{m-n} \operatorname{trace}_{g}\left(B^{\mathscr{V}}\right)^{b}$.

Let $\varphi:\left(M, c_{M}, D^{M}\right) \rightarrow\left(N, c_{N}, D^{N}\right)$ be a horizontally conformal submersion with nowhere degenerate fibres between Weyl spaces. We shall denote, as usual (see, for example, [3]), $\mathscr{V}=\operatorname{ker} d \varphi, \mathscr{H}=\mathscr{V} \perp$. Then, $D^{M}$ and $D^{N}$ induce Weyl partial connections with respect to $\mathscr{V}$ on $\left(\mathscr{H},\left.c_{M}\right|_{\mathscr{H}}\right)$ over $\mathscr{H}$, which will be denoted $\mathscr{H} D^{M}$ and $D^{N}$, respectively. (Recall [26] that a Weyl partial connection $D$ on $(\mathscr{H}, c)$, over $\mathscr{H}$, is a conformal partial connection $D$ on $(\mathscr{H}, c)$ whose torsion tensor field $T$ with respect to $\mathscr{V}$ defined by $T(X, Y)=D_{X} Y-D_{Y} X-\mathscr{H}[X, Y]$ for local sections $X$ and $Y$ of $\mathscr{H}$ is zero.)

If $D$ is a (partial) connection on $L$ and $k \in \mathbb{Z}$, then we shall denote by $D^{k}$ the (partial) connection induced on $L^{k}$, where $L^{k}=\otimes^{k} L$ if $k$ is a natural number and $L^{k}=\otimes^{-k} L^{*}$ if $k$ is a negative integer.

Proposition 3.2. Let $\varphi:\left(M^{m}, c_{M}, D^{M}\right) \rightarrow\left(N^{n}, c_{N}, D^{N}\right)$ be a horizontally conformal submersion with nowhere degenerate fibres between Weyl spaces. Then,

$$
\operatorname{trace}_{c_{M}}(D d \varphi)^{b}=\left(\mathscr{H} D^{M}\right)^{m-2} \otimes\left(D^{N}\right)^{-(n-2)}-\left(D^{\mathrm{Bott}}\right)^{m-n} .
$$

Proof. Let $B^{\mathscr{V}, D^{M}}$ be the second fundamental form of $\mathscr{V}$, with respect to $D^{M}$, defined by

$$
B^{\mathscr{V}, D^{M}}(U, V)=\frac{1}{2} \mathscr{H}\left(D_{U}^{M} V+D_{V}^{M} U\right)
$$

for local sections $U$ and $V$ of $\mathscr{V}$ ([5], cf. [3]). A straightforward calculation gives

$$
\operatorname{trace}_{c_{M}}(D d \varphi)^{b}=\operatorname{trace}_{c_{M}}\left(D^{N}-\mathscr{H} D^{M}\right)-\operatorname{trace}_{c_{M}}\left(B^{\mathscr{V}, D^{M}}\right)^{b} .
$$

Now let $g$ be a local representative of $c_{M}$, corresponding to some local section $s$ of $L$ and let $\alpha_{M}$ and $\alpha_{N}$ be the Lee forms of $D^{M}$ and $D^{N}$, respectively, with respect to $g$. Recall (see [9]) that $\alpha_{M}\left(\alpha_{N}\right)$ is the local connection form of $D^{M}\left(D^{N}\right)$ with respect to $s$. Also, it is easy to prove that

$$
\operatorname{trace}_{g}\left(B^{\mathscr{V}, D^{M}}\right)^{b}=\operatorname{trace}_{g}\left(B^{\mathscr{V}}\right)^{b}-(m-n) \alpha_{M} \mid \mathscr{H},
$$

where $B^{\mathscr{V}}$ is the second fundamental form of $\mathscr{V}$ with respect to (the LeviCivita connection of) $g$. 
It follows that (3.2) is equivalent to

$$
\operatorname{trace}_{c_{M}}(D d \varphi)^{b}=\left.(m-2) \alpha_{M}\right|_{\mathscr{H}}-(n-2) \alpha_{N}-\operatorname{trace}_{g}\left(B^{\mathscr{V}}\right)^{b} .
$$

The proof follows from Example 3.1.

\section{Remark 3.3.}

(1) When $D^{M}$ and $D^{N}$ are the Levi-Civita connections of (local) representatives of $c_{M}$ and $c_{N}$, respectively, then (3.1) reduces to the fundamental equation (see [3]) for horizontally conformal submersions between Riemannian manifolds.

(2) Let $\varphi:\left(M, c_{M}\right) \rightarrow\left(N, c_{N}, D^{N}\right)$ be a horizontally conformal submersion, with nowhere degenerate fibres, from a conformal manifold to a Weyl space. From the fundamental Equation (3.1), it follows that there exists a Weyl connection $D^{M}$ on $\left(M, c_{M}\right)$ such that $\varphi:\left(M, c_{M}, D^{M}\right) \rightarrow$ $\left(N, c_{N}, D^{N}\right)$ is a harmonic morphism.

(3) Another consequence of Proposition 3.2 is that if $D_{1}$ and $D_{2}$ are Weyl connections on a conformal manifold $\left(N, c_{N}\right)$ of dimension not equal to 2 and $\varphi:\left(M, c_{M}, D^{M}\right) \rightarrow\left(N, c_{N}, D_{j}\right), j=1,2$, is a surjective harmonic morphism with nowhere degenerate fibres, then $D_{1}=D_{2}$ (cf. [3]).

We shall say that $\mathscr{V}$ is minimal with respect to $D^{M}$ if $\operatorname{trace}_{c_{M}}\left(B^{\mathscr{V}, D^{M}}\right)=$ 0 ; then $\mathscr{V}$ is minimal with respect to $D^{M}$ if and only if $D^{M}$ and $D^{\text {Bott }}$ induce the same partial connection on $L$ over $\mathscr{H}[5]$.

Similar to the case of harmonic morphisms between Riemannian manifolds, the fundamental Equation (3.1) easily implies the following result.

Theorem 3.4 (cf. [1]). Let $\varphi:\left(M, c_{M}, D^{M}\right) \rightarrow\left(N, c_{N}, D^{N}\right)$ be a horizontally conformal submersion with nowhere degenerate fibres between Weyl spaces.

(a) If $\operatorname{dim} N=2$, then $\varphi$ is a harmonic morphism if and only if its fibres are minimal with respect to $D^{M}$.

(b) If $\operatorname{dim} N \neq 2$, then any two of the following assertions imply the third:

(i) $\varphi$ is a harmonic morphism.

(ii) The fibres of $\varphi$ are minimal with respect to $D^{M}$.

(iii) $\mathscr{H} D^{M}=D^{N}$.

Let $\left(M, c_{M}\right)$ be a conformal manifold endowed with a conformal foliation $\mathscr{V}$. Note that if $\mathscr{V}$ is nowhere degenerate, then $L$ is basic (with respect to 
$\mathscr{V})$ [5]. Indeed, for any point $x \in M$, there exists an open neighbourhood $U$, of $x$, on which there can be defined a horizontally conformal submersion $\varphi:\left(U,\left.c_{M}\right|_{U}\right) \rightarrow\left(N, c_{N}\right)$ which defines $\mathscr{V}$ on $U$ and which admits a dilation $\lambda$. Then $\lambda$ is an isomorphism between $\left.L\right|_{U}$ and $\varphi^{*}\left(L_{N}\right)$.

The following proposition follows easily from (3.1).

Proposition 3.5 (cf. [3]). Let $\left(M^{m}, c_{M}, D^{M}\right)$ be a Weyl space, $\operatorname{dim} M=$ $m$, endowed with a nowhere degenerate conformal foliation $\mathscr{V}$.

(i) If $\operatorname{codim} \mathscr{V}=2$, then $\mathscr{V}$ produces harmonic morphisms on $\left(M, c_{M}, D^{M}\right)$ if and only if its leaves are minimal.

(ii) If $\operatorname{codim} \mathscr{V}=n \neq 2$, then $\mathscr{V}$ produces harmonic morphisms on $\left(M, c_{M}\right.$, $\left.D^{M}\right)$ if and only if the partial connection $\left(\mathscr{H} D^{M}\right)^{m-2} \otimes\left(D^{\mathrm{Bott}}\right)^{-(m-n)}$ on $L^{n-2}$, over $\mathscr{H}$, is basic.

We end this section with an example of a Weyl connection which will be useful later on.

Example 3.6 [5]. Let $\left(M^{m}, c\right)$ be a conformal manifold endowed with a nondegenerate distribution $\mathscr{V}$ of codimension $n$ and let $\mathscr{H}=\mathscr{V}^{\perp}$.

For each local representative $g$ of $c_{M}$, define a (local) 1-form $\alpha^{g}$ by

$$
\alpha^{g}=\frac{1}{m-n} \operatorname{trace}_{g}\left(B^{\mathscr{V}}\right)^{b}+\frac{1}{n} \operatorname{trace}_{g}\left(B^{\mathscr{H}}\right)^{b} .
$$

Then $\alpha^{g \lambda^{-2}}=\alpha^{g}+\lambda^{-1} d \lambda$. Hence, the family of 1-forms $\left\{\alpha^{g}\right\}$ defines a Weyl connection $D$ on $\left(M^{m}, c\right)$. The Weyl connection $D$ is called the (minimal) Weyl connection of $\left(M^{m}, c, \mathscr{V}\right)$. Note that if we denote by $D^{\text {Bott, } \mathscr{V}}$ and $D^{\text {Bott, } \mathscr{H}}$ the partial connections over $\mathscr{H}$ and $\mathscr{V}$, respectively, induced on $L$ by the Bott partial connections (see Example 3.1) on $\mathscr{V}$ and $\mathscr{H}$, then $D=D^{\text {Bott }, \mathscr{V}}+D^{\text {Bott, } \mathscr{H}}$. Also, note that $\operatorname{trace}_{c}(D \mathscr{V})=\operatorname{trace}_{c}(D \mathscr{H})=0$ (cf. Remark 4.7 below).

As (3.3) holds without the assumption that $\mathscr{V}$ is conformal, $\mathscr{V}$ and $\mathscr{H}$ are minimal with respect to $D$; moreover, $D$ is the unique Weyl connection on $\left(M^{m}, c\right)$ with this property. It follows that if $\mathscr{V}$ is one-dimensional and conformal, then the connection induced by $D$ on $L$ is flat if and only if $\mathscr{V}$ is locally generated by conformal vector fields. 


\section{Harmonic maps and morphisms between almost Hermitian manifolds}

In this section, we shall work in the smooth and (real or complex) analytic categories. An almost Hermitian (conformal) manifold is a triple $(M, c, J)$, where $(M, c)$ is a conformal manifold and $J$ is a compatible almost complex structure; that is, if we consider $c$ as an $L^{2}$-valued Riemannian metric on $M$ [9], then we have $c(J X, J Y)=c(X, Y),(X, Y \in T M)$. Therefore, $\operatorname{dim} M$ is even and the Kähler form of $(M, c, J)$, defined by $\omega(X, Y)=c(J X, Y)$, $(X, Y \in T M)$, is an $L^{2}$-valued almost symplectic structure on $M$. A Hermitian (conformal) manifold is an almost Hermitian manifold $(M, c, J)$ such that $J$ is integrable.

To any almost Hermitian manifold, of dimension at least 4, can be associated, in a natural way, a Weyl connection as follows.

Proposition 4.1 [28]. Let $(M, c, J)$ be an almost Hermitian manifold of dimension $m \geq 4$, and let $\omega \in \Gamma\left(L^{2} \otimes \Lambda^{2} T^{*} M\right)$ be its Kähler form.

There exists a unique Weyl connection $D$ on $(M, c)$ such that $\operatorname{trace}_{c}(D J)=0$; the Lee form of $D$ with respect to a local representative $g$ of $c$ is equal to $-1 /(m-2)$ times the Lee form of $J$ with respect to $g$.

Proof. Let $m=2 n,(n \geq 2)$. From the fact that $\omega$ is an $L^{2}$-valued almost symplectic structure on $M$, it follows [5] that there exists a unique connection $D$ on $L^{2}$ such that

$$
d^{D} \omega \wedge \omega^{n-2}=0
$$

We shall denote by the same letter $D$ the induced connection on $L$ and the corresponding Weyl connection on $(M, c)$. Let $s$ be a local section of $L$ and let $\omega^{s}$ be the Kähler form of $J$ with respect to the local representative $g^{s}$ of $c$ corresponding to $s$; that is, $\omega^{s}(X, Y)=g^{s}(J X, Y),(X, Y \in T M)$. It is easy to prove that (4.1) is equivalent to the fact that for any local section $s$ of $L$, the local connection form of $D$ with respect to $s$ is equal to $-1 /(m-2)$ times the Lee form of $J$ with respect to $g^{s}$.

Furthermore, (4.1) is also equivalent to $\sum_{i=1}^{n}\left(d^{D} \omega\right)\left(X_{i}, J X_{i}, \cdot\right)=0$ for any conformal frame $\left\{X_{1}, J X_{1}, \ldots, X_{n}, J X_{n}\right\}$. Therefore, to end the proof, it is sufficient to show that for any Weyl connection $D$ on $(M, c)$, we have

$$
\sum_{i=1}^{n}\left(d^{D} \omega\right)\left(X_{i}, J X_{i}, J Y\right)=-c\left(\operatorname{trace}_{g}(D J), Y\right)
$$


for any $Y \in T M$, and where $g$ is the metric determined by the conformal frame $\left\{X_{1}, J X_{1}, \ldots, X_{n}, J X_{n}\right\}$. Indeed, as $D c=0$, we have $(D \omega)(X, Y)=$ $c((D J)(X), Y)$ and $(D \omega)(X, J X)=0,(X, Y \in T M)$. Therefore,

$$
\begin{aligned}
\left(d^{D} \omega\right)\left(X_{i}, J X_{i}, J Y\right)= & \left(D_{X_{i}} \omega\right)\left(J X_{i}, J Y\right)+\left(D_{J X_{i}} \omega\right)\left(J Y, X_{i}\right) \\
& +\left(D_{J Y} \omega\right)\left(X_{i}, J X_{i}\right) \\
= & c\left(\left(D_{X_{i}} J\right)\left(J X_{i}\right), J Y\right)+c\left(\left(D_{J X_{i}} J\right)(J Y), X_{i}\right) \\
= & -c\left(J\left(D_{X_{i}} J\right)\left(X_{i}\right), J Y\right)-c\left(J\left(D_{J X_{i}} J\right)(Y), X_{i}\right) \\
= & -c\left(\left(D_{X_{i}} J\right)\left(X_{i}\right), Y\right)-c\left(Y,\left(D_{J X_{i}} J\right)\left(J X_{i}\right)\right)
\end{aligned}
$$

and the proof follows.

Definition $4.2[28]$. Let $(M, c, J)$ be an almost Hermitian manifold of dimension $\operatorname{dim} M \geq 4$.

The Weyl connection of $(M, c, J)$ is the Weyl connection $D$ on $(M, c)$ such that $\operatorname{trace}_{c}(D J)=0$.

\section{Remark 4.3.}

(1) [28] Let $(M, c, J)$ be an almost Hermitian manifold of dimension dim $M \geq 4$, and let $D$ be a Weyl connection on $(M, c)$.

Let $\nabla$ be the Levi-Civita connection of a local representative $g$ of c. Then, $D_{J X} J-J D_{X} J=\nabla_{J X} J-J \nabla_{X} J,(X \in T M)$. Hence, $J$ is integrable if and only if $D_{J X} J=J D_{X} J,(X \in T M)$.

On the other hand, the condition $D_{J X} J=-J D_{X} J,(X \in T M)$, is equivalent to $\left(d^{D} \omega\right)^{(1,2) \oplus(2,1)}=0$ and is a sufficient condition for $D$ to be The Weyl connection of $(M, c, J)$. Hence, if $\operatorname{dim} M=4$, then $D_{J X} J=-J D_{X} J,(X \in T M)$, if and only if $D$ is the Weyl connection of $(M, c, J)$.

Thus, if $\operatorname{dim} M=4$, then $D J=0$ if and only if $J$ is integrable and $D$ is the Weyl connection of $(M, c, J)$. If $\operatorname{dim} M \geq 6$, then it follows that $D J=0$ if and only if, locally, there exist representatives $g$ of $c$ with respect to which $(M, g, J)$ is Kähler.

(2) Let $(M, c, J)$ be an almost Hermitian manifold and let $f$ be a $( \pm)$ holomorphic function locally defined on $(M, J)$. (If $(M, c, J)$ is complex analytic, then by a $( \pm)$ holomorphic function, we mean a function which is constant along curves tangent to the $(\mp \mathrm{i})$ eigendistributions of $J$.) If $\operatorname{dim} M=2$, then $f$ is harmonic with respect to any local 
representative of $c$ (see [3]). If $\operatorname{dim} M \geq 4$ and $D$ is the Weyl connection of $(M, c, J)$, then $f$ is a harmonic function of $(M, c, D)$.

Furthermore, if $(M, c, J)$ is a Hermitian manifold, $\operatorname{dim} M \geq 4$, then for any Weyl connection $D$ on $(M, c)$, the following assertions are equivalent:

(i) $D$ is the Weyl connection of $(M, c, J)$.

(ii) Any $( \pm)$ holomorphic function of $(M, J)$ is a harmonic function of $(M, c, D)$.

See Proposition 4.8 for a reformulation of this equivalence in the complex analytic category.

Next, we prove the following useful lemma.

Lemma 4.4 (cf. $[18,24]$ ). Let $D^{M}, D^{N}$ be torsion-free connections on the almost complex manifolds $\left(M, J^{M}\right),\left(N, J^{N}\right)$, respectively. Suppose that $M$ is endowed with a conformal structure $c$ and let $\varphi:\left(M, J^{M}\right) \rightarrow\left(N, J^{N}\right)$ be a holomorphic map. Then,

$$
\operatorname{trace}_{c} \varphi^{*}\left(D^{N} J^{N}\right)-d \varphi\left(\operatorname{trace}_{c}\left(D^{M} J^{M}\right)\right)+J^{N}\left(\operatorname{trace}_{c}(D d \varphi)\right)=0 .
$$

Proof. It is easy to prove that for $X, Y \in T M$, we have

$$
\begin{aligned}
D d \varphi\left(X, J^{M} Y\right)= & \left(D_{d \varphi(X)}^{N} J^{N}\right)(d \varphi(Y))-d \varphi\left(\left(D_{X}^{M} J^{M}\right)(Y)\right) \\
& +J^{N}(D d \varphi(X, Y)) .
\end{aligned}
$$

The proof follows.

From Lemma 4.4, we easily obtain the following proposition (cf. Remark $4.3(2))$.

Proposition 4.5 (cf. $[18,12])$. Let $\left(M, c_{M}, J^{M}\right)$ and $\left(N, c_{N}, J^{N}\right)$ be almost Hermitian manifolds. If $\operatorname{dim} M \geq 4$, $\operatorname{dim} N \geq 4$, let $D^{M}, D^{N}$ be the Weyl connections of $\left(M, c_{M}, J^{M}\right),\left(N, c_{N}, J^{N}\right)$, respectively; if $\operatorname{dim} M=2$ or $\operatorname{dim} N=$ 2 , then $D^{M}$ or $D^{N}$ will denote any Weyl connection on $\left(M, c_{M}\right)$ or $\left(N, c_{N}\right)$, respectively.

Let $\varphi:\left(M, J^{M}\right) \rightarrow\left(N, J^{N}\right)$ be a holomorphic map.

(i) If $\left(d^{D^{N}} \omega_{N}\right)^{(1,2) \oplus(2,1)}=0$, where $\omega_{N}$ is the Kähler form of $\left(N, c_{N}, J^{N}\right)$, then $\varphi:\left(M, c_{M}, D^{M}\right) \rightarrow\left(N, c_{N}, D^{N}\right)$ is a harmonic map. 
(ii) If the map $\varphi:\left(M, c_{M}\right) \rightarrow\left(N, c_{N}\right)$ is horizontally weakly conformal and has nowhere degenerate fibres, then $\varphi:\left(M, c_{M}, D^{M}\right) \rightarrow\left(N, c_{N}, D^{N}\right)$ is a harmonic map and hence a harmonic morphism.

Note that in assertion (i) of Proposition 4.5, if $\operatorname{dim} N=2,4$, then the condition $\left(d^{D^{N}} \omega_{N}\right)^{(1,2) \oplus(2,1)}=0$ is automatically satisfied. Also, in (ii) of Proposition 4.5, we automatically have $\varphi$ horizontally weakly conformal if $\operatorname{dim} N=2$. Therefore, we have the following result.

Corollary 4.6. Any holomorphic map from an almost Hermitian manifold, endowed with its Weyl connection, to a two-dimensional oriented conformal manifold is a harmonic morphism.

The Weyl connections of Example 3.6 and Definition 4.2 can be generalized as follows.

Remark 4.7. Let $(M, c)$ be a conformal manifold, $\operatorname{dim} M=m$, endowed with a section $P$ of $\operatorname{End}(T M)$ and let $\mathcal{P}=(m-1) P+P^{*}-\operatorname{trace}(P) I d_{T M}$. It is easy to prove that if $\mathcal{P}$ is invertible at each point, then for any 1 -form $\alpha$ on $M$, there exists a unique Weyl connection $D$ on $(M, c)$ such that $\operatorname{trace}_{c}(D P)^{b}=\alpha$.

Sufficient conditions under which $\mathcal{P}$ is invertible at each point are as follows:

(a) $P$ is self-adjoint and $\frac{1}{m} \operatorname{trace}(P)$ is not an eigenvalue of $P$ (for example, if $P=\mathscr{V}$ of Example 3.6).

(b) $P$ is skew-adjoint, invertible at each point and $m \geq 3$ (for example, if $P=J$ of Definition 4.2).

Furthermore, Proposition 4.5(ii) can be easily generalized to the case when $P$ satisfies condition (b).

The result of Proposition 4.5(ii) can be extended as follows.

Proposition 4.8. Let $\left(M, c_{M}, J^{M}\right)$ be a complex analytic almost Hermitian manifold. If $\operatorname{dim} M \geq 4$, let $D^{M}$ be the Weyl connection of $\left(M, c_{M}, J^{M}\right)$; if $\operatorname{dim} M=2$, let $D^{M}$ be any Weyl connection on $\left(M, c_{M}\right)$.

Let $\varphi:\left(M, c_{M}\right) \rightarrow N$ be a horizontally conformal submersion with degenerate fibres such that ker $d \varphi$ contains $\mathscr{F}$ or $\widetilde{\mathscr{F}}$, where $\mathscr{F}, \widetilde{F}$ are the eigendistributions of $J^{M}$. 
Then, $\varphi:\left(M, c_{M}, D^{M}\right) \rightarrow\left(N, D^{N}\right)$ is a harmonic map with respect to any connection $D^{N}$ on $N$, and $\varphi:\left(M, c_{M}, D^{M}\right) \rightarrow\left(N, c_{N}, D^{N}\right)$ is a harmonic morphism with respect to any structure of Weyl space on $N$.

Conversely, if $\operatorname{dim} M \geq 4, J^{M}$ is integrable and $D$ is a Weyl connection on $\left(M, c_{M}\right)$ such that the foliations $\mathscr{F}$ and $\widetilde{F}$ are locally defined by harmonic maps with respect to $c_{M}, D$, then $D$ is the Weyl connection of $\left(M, c_{M}, J^{M}\right)$.

Proof. Suppose that $\mathscr{F} \subseteq$ ker $d \varphi$. Then for any function $f$, locally defined on $N$, the function $f \circ \varphi$ is a holomorphic function of $\left(M, J^{M}\right)$. By Remark 4.3(2), $f \circ \varphi$ is a harmonic function of $\left(M, c_{M}, D^{M}\right)$ and hence $\varphi:\left(M, c_{M}, D^{M}\right) \rightarrow\left(N, c_{N}, D^{N}\right)$ is a harmonic morphism with respect to any structure of Weyl space on $N$.

The second statement follows from the implication (ii) $\Rightarrow$ (i) of Remark $4.3(2)$.

Let $\left(N^{2}, c_{N}\right)$ be a two-dimensional orientable conformal manifold. Then, there exists a complex structure $J^{N}$, uniquely determined up to sign, with respect to which $\left(N^{2}, c_{N}, J^{N}\right)$ is a Hermitian manifold.

Let $\left(M^{4}, c_{M}\right)$ be a four-dimensional complex analytic oriented conformal manifold. An (anti-) self-dual space at $x \in M$ is a two-dimensional vector space $p \subseteq T_{x} M$ such that for some (and hence any) basis $\{X, Y\}$ of $p$, the 2-form $X \wedge Y$ is (anti-)self-dual; if $\left(M^{4}, c_{M}\right)$ is an oriented smooth or real analytic manifold, then an (anti-)self-dual space at $x \in M$ is an (anti-)selfdual subspace of $\left(T_{x}^{\mathbb{C}} M,\left(c_{M}\right)_{x}^{\mathbb{C}}\right)$ (see [21]).

Let $\left(M^{4}, c_{M}\right)$ be a four-dimensional oriented conformal manifold endowed with a two-dimensional nondegenerate distribution $\mathscr{V}$. Suppose, for simplicity, that $\mathscr{V}$ (and hence also its orthogonal complement $\mathscr{H}$ ) endowed with the conformal structure induced by $c_{M}$ is orientable. We choose orientations on $\left(\mathscr{V},\left.c_{M}\right|_{\mathscr{V}}\right)$ and $\left(\mathscr{H},\left.c_{M}\right|_{\mathscr{H}}\right)$, so that a conformal frame $\left(X_{1}, \ldots, X_{4}\right)$ on $\left(M^{4}, c_{M}\right)$, adapted to the orthogonal decomposition $T M=\mathscr{V} \oplus \mathscr{H}$, to be positive if $\left(X_{1}, X_{2}\right)$ and $\left(X_{3}, X_{4}\right)$ are positively oriented frames on $\left(\mathscr{V},\left.c_{M}\right|_{\mathscr{V}}\right)$ and $\left(\mathscr{H}, c_{M} \mid \mathscr{H}\right)$, respectively. Then there exists an almost complex structure $J^{M}$, uniquely determined up to sign, with respect to which $\left(M^{4}, c_{M}, J^{M}\right)$ is a positive almost Hermitian manifold such that $J^{M}(\mathscr{V})=\mathscr{V}$. (We say that $\left(M^{4}, c_{M}, J^{M}\right)$ is positive if some (and hence, any) conformal frame of the form $\left(X_{1}, J^{M} X_{1}, X_{2}, J^{M} X_{2}\right)$ is positive; equivalently, at some (and hence, any) point, the eigenspaces of $J^{M}$ are self-dual. Note that in the smooth and real analytic categories, this just means that $J^{M}$ is a positive almost complex structure on $M^{4}$.) It follows that the Weyl connection of $\left(M^{4}, c_{M}, J^{M}\right)$ 
is equal to

$$
D-\frac{1}{2}\left(J^{M}\left(* \mathscr{V} I^{\mathscr{V}}\right)\right)^{b}-\frac{1}{2}\left(J^{M}\left(* \mathscr{H} I^{\mathscr{H}}\right)\right)^{b},
$$

where $D$ is the Weyl connection of $\left(M^{4}, c_{M}, \mathscr{V}\right), I^{\mathscr{V}}, I^{\mathscr{H}}$ are the integrability tensors of $\mathscr{V}, \mathscr{H}$, respectively, $*_{\mathscr{V}}, *_{\mathscr{H}}$ are the Hodge star-operators of $\left(\mathscr{V},\left.c_{M}\right|_{\mathscr{V}}\right)$ and $\left(\mathscr{H},\left.c_{M}\right|_{\mathscr{H}}\right)$, respectively and ${ }^{b}: T M \otimes L_{M}^{*} \longrightarrow T^{*} M$ is the "musical" isomorphism of $\left(M^{4}, c_{M}\right)$ [5]; equivalently, the Lee form of $J^{M}$ with respect to any local representative $g$ of $c_{M}$ is equal to

$$
-\operatorname{trace}_{g}\left(B^{\mathscr{V}}\right)^{b}-\operatorname{trace}_{g}\left(B^{\mathscr{H}}\right)^{b}+\left(J^{M}\left(* \mathscr{V} I^{\mathscr{V}}\right)\right)^{b}+\left(J^{M}\left(* \mathscr{H} I^{\mathscr{H}}\right)\right)^{b} .
$$

Let $\varphi:\left(M^{4}, c_{M}\right) \rightarrow\left(N^{2}, c_{N}\right)$ be a horizontally conformal submersion, with nowhere degenerate fibres, between oriented conformal manifolds of dimensions 4 and 2. Then, there exists a unique almost complex structure $J^{M}$ on $M^{4}$ with respect to which the map $\varphi:\left(M^{4}, J^{M}\right) \rightarrow\left(N^{2}, J^{N}\right)$ is holomorphic and $\left(M^{4}, c_{M}, J^{M}\right)$ is a positive almost Hermitian manifold. Let $D^{M}$ be a Weyl connection on $\left(M^{4}, c_{M}\right)$.

Proposition 4.9 (cf. [29]). The following assertions are equivalent:

(i) The map $\varphi:\left(M^{4}, c_{M}, D^{M}\right) \rightarrow\left(N^{2}, c_{N}\right)$ is a harmonic morphism and $J^{M}$ is integrable.

(ii) The almost complex structure $J^{M}$ is parallel along the fibres of $\varphi$ with respect to $D^{M}$; that is, $D_{U}^{M} J^{M}=0,(U \in \operatorname{ker} d \varphi)$.

Proof. First, we shall write the proof in the complex analytic category. Let $\mathscr{F}$ and $\widetilde{F}$ be the eigendistributions of $J^{M}$. Then, $J^{M}$ is integrable if and only if $\mathscr{F}$ and $\widetilde{\mathscr{F}}$ are integrable. Also note that assertion (ii) holds if and only if $\mathscr{F}$ and $\widetilde{F}$ are parallel with respect to $D^{M}$ along $\mathscr{V}(=\operatorname{ker} d \varphi)$.

Let $f$ and $\widetilde{f}$ be the components of $\varphi$ with respect to null local coordinates on $\left(N^{2}, c_{N}\right)$. As $\varphi:\left(M^{4}, c_{M}\right) \rightarrow\left(N^{2}, c_{N}\right)$ is horizontally conformal, $\varphi:\left(M^{4}, c_{M}, D^{M}\right) \rightarrow\left(N^{2}, c_{N}\right)$ is a harmonic morphism if and only if $f$ and $\tilde{f}$ are harmonic functions of $\left(M^{4}, c_{M}, D^{M}\right)$. Also, we may suppose $\mathscr{F} \subseteq$ ker $d f$, $\widetilde{\mathscr{F}} \subseteq \operatorname{ker} d \tilde{f}$.

There exists a local frame $\{U, \widetilde{U}, Y, \tilde{Y}\}$ on $M^{4}$ such that $g=2(U \odot \widetilde{U}+$ $Y \odot \widetilde{Y})$ is a local representative of $c_{M}, U, \widetilde{U}$ are vertical, $Y, \widetilde{Y}$ are horizontal and $\{U, Y\},\{\widetilde{U}, \widetilde{Y}\}$ are local frames of $\mathscr{F}, \widetilde{\mathscr{F}}$, respectively.

As $\{U, \widetilde{U}, Y\}$ is a local frame of ker $d f$, we have $g([U, Y], Y)=0$. Hence, $\mathscr{F}$ is integrable if and only if $g([U, Y], U)=0$. As $g([U, Y], U)=g\left(D_{U}^{M} Y, U\right)$, we obtain that $\mathscr{F}$ is integrable if and only if $\mathscr{F}$ is parallel along $U$, with respect to $D^{M}$. 
Also, $\operatorname{trace}_{g}\left(D^{M} d f\right)=-2 g\left(D_{U}^{M} \widetilde{U}, Y\right) \widetilde{Y}(f)$. Hence, $f$ is a harmonic function of $\left(M^{4}, c_{M}, D^{M}\right)$ if and only if $\mathscr{F}$ is parallel along $\widetilde{U}$, with respect to $D^{M}$.

Therefore, $\mathscr{F}$ is integrable and $f$ is harmonic if and only if $\mathscr{F}$ is parallel, with respect to $D^{M}$, along $\mathscr{V}$. Similarly, $\widetilde{\mathscr{F}}$ is integrable and $\widetilde{f}$ is harmonic if and only if $\widetilde{\mathscr{F}}$ is parallel with respect to $D^{M}$ along $\mathscr{V}$. Thus, the proof is complete in the complex analytic category.

In the smooth and real analytic categories, essentially the same argument applies to the complexification $(d \varphi)^{\mathbb{C}}: T^{\mathbb{C}} M \rightarrow T^{\mathbb{C}} N$.

\section{Harmonic morphisms and twistorial maps}

In this section, we shall work in the complex analytic category.

We continue the study, initiated in the previous section, of the relation between harmonic morphisms and twistorial maps. We start with a brief presentation of the examples of twistorial maps with which we shall work; more details can be found in [26] (and in [20], for the notions of almost twistorial structure and twistorial map in the smooth category).

Example 5.1. Let $\left(M^{3}, c_{M}, D^{M}\right)$ be a three-dimensional Weyl space and let $\left(N^{2}, c_{N}\right)$ be a two-dimensional conformal manifold.

A twistorial map $\varphi:\left(M^{3}, c_{M}, D^{M}\right) \rightarrow\left(N^{2}, c_{N}\right)$ with nowhere degenerate fibres is a horizontally conformal submersion whose fibres are geodesics with respect to $D^{M}$. The existence of such twistorial maps is related to $\left(M^{3}, c_{M}, D^{M}\right)$ being Einstein-Weyl [13] (see [26]; see also Remark 6.4(1) below).

Let $\varphi: M^{3} \rightarrow N^{2}$ be a submersion with nowhere degenerate fibres and let $p, \widetilde{p}$ be the two-dimensional degenerate distributions locally defined on $\left(M^{3}, c_{M}\right)$ such that ker $d \varphi=p \cap \widetilde{p}$. Then, $\varphi:\left(M^{3}, c_{M}\right) \rightarrow\left(N^{2}, c_{N}\right)$ is horizontally conformal if and only if $p$ and $\widetilde{p}$ are integrable. It follows that $\varphi:\left(M^{3}, c_{M}, D^{M}\right) \rightarrow\left(N^{2}, c_{N}\right)$ is twistorial if and only if $p$ and $\widetilde{p}$ are integrable and their integral manifolds are totally geodesic with respect to $D^{M}$; note that $\varphi$ maps any such surface to a null geodesic on $\left(N^{2}, c_{N}\right)$.

By Theorems 2.9 and $3.4, \varphi:\left(M^{3}, c_{M}, D^{M}\right) \rightarrow\left(N^{2}, c_{N}\right)$ is a twistorial map if and only if it is a harmonic morphism.

Example 5.2. Let $\left(M^{4}, c_{M}\right)$ and $\left(N^{2}, c_{N}\right)$ be oriented conformal manifolds of dimensions 4 and 2 , respectively.

A twistorial map $\varphi:\left(M^{4}, c_{M}\right) \rightarrow\left(N^{2}, c_{N}\right)$ with nowhere degenerate fibres is a horizontally conformal submersion for which the almost complex structure $J^{M}$ on $M^{4}$, with respect to which $\varphi:\left(M^{4}, J^{M}\right) \rightarrow\left(N^{2}, J^{N}\right)$ is 
holomorphic and $\left(M^{4}, c_{M}, J^{M}\right)$ is a positive almost Hermitian manifold, is integrable (cf. [29]).

If $\varphi:\left(M^{4}, c_{M}, D^{M}\right) \rightarrow\left(N^{2}, c_{N}\right)$ is twistorial and $\mathscr{F}, \widetilde{F}$ are the, necessarily integrable, eigendistributions of $J^{M}$, then $\varphi$ maps the leaves of $\mathscr{F}$ and $\widetilde{F}$ to null geodesics on $\left(N^{2}, c_{N}\right)$.

By Remark 4.3(1), $\varphi:\left(M^{4}, c_{M}\right) \rightarrow\left(N^{2}, c_{N}\right)$ is a twistorial map if and only if $D^{M} J^{M}=0$, where $D^{M}$ is the Weyl connection of $\left(M^{4}, c_{M}, J^{M}\right)$. Furthermore, if $\varphi:\left(M^{4}, c_{M}\right) \rightarrow\left(N^{2}, c_{N}\right)$ is twistorial, then, by Proposition 4.5, $\varphi:\left(M^{4}, c_{M}, D^{M}\right) \rightarrow\left(N^{2}, c_{N}\right)$ is a harmonic morphism. More generally, by Proposition 4.9 , if $D$ is a Weyl connection on $\left(M^{4}, c_{M}\right)$, then $\varphi:\left(M^{4}, c_{M}, D\right) \rightarrow\left(N^{2}, c_{N}\right)$ is a twistorial harmonic morphism if and only if $J^{M}$ is parallel along the fibres of $\varphi$ with respect to $D$.

A two-dimensional foliation $\mathscr{V}$ with nowhere degenerate leaves on $\left(M^{4}\right.$, $\left.c_{M}\right)$ is twistorial if it can be locally defined by twistorial maps; note that $\mathscr{V}$ is twistorial with respect to both orientations of $\left(M^{4}, c_{M}\right)$ if and only if its leaves are totally umbilical. If $\left(M^{4}, c_{M}\right)$ is nonorientable, then $\mathscr{V}$ is twistorial if its lift to the oriented $\mathbb{Z}_{2}$-covering space of $\left(M^{4}, c_{M}\right)$ is twistorial; equivalently, $\mathscr{V}$ has totally umbilical leaves.

Example 5.3. Let $\left(M^{4}, c_{M}\right)$ be an oriented four-dimensional conformal manifold, and let $\left(N^{3}, c_{N}, D^{N}\right)$ be a three-dimensional Weyl space.

Let $\varphi:\left(M^{4}, c_{M}\right) \rightarrow\left(N^{3}, c_{N}\right)$ be a horizontally conformal submersion with nowhere degenerate fibres. Let $\mathscr{V}=\operatorname{ker} d \varphi, \mathscr{H}=\mathscr{V}^{\perp}$ and let $D$ be the Weyl connection of $\left(M^{4}, c_{M}, \mathscr{V}\right)$ (see Example 3.6). Let $I^{\mathscr{H}}$ be the integrability tensor of $\mathscr{H}$ defined by $I^{\mathscr{H}}(X, Y)=-\mathscr{V}[X, Y]$ for any horizontal local vector fields $X$ and $Y$.

As both $\mathscr{V}$ and $\mathscr{H}$ are distributions of odd dimensions, the orientation of $\left(M^{4}, c_{M}\right)$ corresponds to an isomorphism between the line bundles canonically associated to the conformal structures induced by $c_{M}$ on $\mathscr{V}$ and $\mathscr{H}$. Hence, as $\mathscr{V}$ is one dimensional, both these line bundles are isomorphic to $\mathscr{V}$. Therefore, if we apply the Hodge star-operator $* \mathscr{H}$ of $\left(\mathscr{H},\left.c_{M}\right|_{\mathscr{H}}\right)$ to the integrability tensor $I^{\mathscr{H}} \in \Gamma\left(\mathscr{V} \otimes \Lambda^{2} \mathscr{H}^{*}\right)$ of $\mathscr{H}$, we obtain a horizontal 1-form on $M^{4}$. Let $D_{ \pm}$be the Weyl partial connections on $\left(\mathscr{H}, c_{M} \mid \mathscr{H}\right)$, over $\mathscr{H}$, given by $D_{ \pm}=\mathscr{H} D \pm * \mathscr{H} I^{\mathscr{H}}([5]$, see $[26])$.

The map $\varphi:\left(M^{4}, c_{M}\right) \rightarrow\left(N^{3}, c_{N}, D^{N}\right)$ is twistorial, with respect to the given orientation on $\left(M^{4}, c_{M}\right)$, if and only if it is horizontally conformal and the Weyl partial connection on $\left(\mathscr{H}, c_{M} \mid \mathscr{H}\right)$, over $\mathscr{H}$, determined by $D^{N}$ is equal to $D_{+}$.

The following assertions are equivalent for a submersion $\varphi: M^{4} \rightarrow N^{3}$ with connected nowhere degenerate fibres ([5], see [26]): 
(i) There exists a Weyl connection $D^{N}$ on $\left(N^{3}, c_{N}\right)$ with respect to which $\varphi:\left(M^{4}, c_{M}\right) \rightarrow\left(N^{3}, c_{N}, D^{N}\right)$ is twistorial.

(ii) $\varphi:\left(M^{4}, c_{M}\right) \rightarrow\left(N^{3}, c_{N}\right)$ is horizontally conformal and the curvature form of the connection induced by $D$ on $L_{M}$ is anti-self-dual (that is, $\varphi:\left(M^{4}, c_{M}\right) \rightarrow\left(N^{3}, c_{N}, D^{N}\right)$ is anti-self-dual in the sense of [5]).

If $\left(M^{4}, c_{M}\right)$ is anti-self-dual, then the following assertions can be added to this list [5] (cf. $[13,15]$; see [26]).

(iii) There exists an Einstein-Weyl connection $D^{N}$ on $\left(N^{3}, c_{N}\right)$ such that for any twistorial map $\psi$ locally defined on $\left(N^{3}, c_{N}, D^{N}\right)$ with values in a conformal manifold $\left(P^{2}, c_{P}\right)$, the map $\psi \circ \varphi$ from $\left(M^{4}, c_{M}\right)$ to $\left(P^{2}, c_{P}\right)$ is twistorial.

(iv) There exists an Einstein-Weyl connection $D^{N}$ on $\left(N^{3}, c_{N}\right)$ such that $\varphi$ maps self-dual surfaces on $\left(M^{4}, c_{M}\right)$ to degenerate surfaces on $\left(N^{3}\right.$, $\left.c_{N}\right)$, which are totally geodesic with respect to $D^{N}$.

It follows that if $\varphi:\left(M^{4}, c_{M}\right) \rightarrow\left(N^{3}, c_{N}, D^{N}\right)$ is twistorial, then $\left(M^{4}\right.$, $\left.c_{M}\right)$ is anti-self-dual if and only if $\left(N^{3}, c_{N}, D^{N}\right)$ is Einstein-Weyl [5] (cf. $[13,15]$; see [26]). Furthermore, if $\left(M^{4}, c_{M}\right)$ is anti-self-dual, then, locally, $\varphi$ corresponds to a submersion $Z(\varphi)$ from the twistor space $Z(M)$ of $\left(M^{4}, c_{M}\right)$ onto the twistor space $Z(N)$ of $\left(N^{3}, c_{N}, D^{N}\right)$, which maps each twistor line on $Z(M)$ diffeomorphically onto a twistor line on $Z(N)$; the map $Z(\varphi)$ is the twistorial representation of $\varphi$.

As in Example 5.2, a one-dimensional foliation $\mathscr{V}$ with nowhere degenerate leaves is twistorial if it can be locally defined by twistorial maps. Note that $\mathscr{V}$ is twistorial with respect to both orientations of $\left(M^{4}, c_{M}\right)$ if and only if it is locally generated by conformal vector fields [5] (this follows from the equivalence (i) $\Longleftrightarrow$ (ii) above and Example 3.6). If $\left(M^{4}, c_{M}\right)$ is nonorientable, then $\mathscr{V}$ is twistorial if its lift to the oriented $\mathbb{Z}_{2}$-covering space of $\left(M^{4}, c_{M}\right)$ is twistorial; equivalently, $\mathscr{V}$ is locally generated by nowhere zero conformal vector fields.

Next, we give necessary and sufficient conditions under which a map between Weyl spaces of dimensions 4 and 3 is a twistorial harmonic morphism.

Theorem 5.4. Let $\left(M^{4}, c_{M}, D^{M}\right)$ be an oriented Weyl space of dimension 4 and let $\left(N^{3}, c_{N}\right)$ be a conformal manifold of dimension 3. We denote by $\mathscr{D}^{M} \subseteq T P_{M}$ the connection induced by $D^{M}$ on the bundle $\pi_{M}: P_{M} \rightarrow M$ of 
self-dual spaces on $\left(M^{4}, c_{M}\right)$. Also, we denote by $\pi_{N}: P_{N} \rightarrow N$ the bundle of two-dimensional degenerate spaces on $\left(N^{3}, c_{N}\right)$.

Let $\varphi:\left(M^{4}, c_{M}\right) \rightarrow\left(N^{3}, c_{N}\right)$ be a horizontally conformal submersion with connected nowhere degenerate fibres and let $D$ be the Weyl connection of $\left(M^{4}, c_{M}, \mathscr{V}\right)$. We denote by $\Phi: P_{M} \rightarrow P_{N}$ the bundle map defined by $\Phi(p)=d \varphi(p),\left(p \in P_{M}\right)$.

(a) Let $D^{N}$ be a Weyl connection on $\left(N^{3}, c_{N}\right)$. Any two of the following assertions imply the third:

(a1) $\varphi:\left(M^{4}, c_{M}, D^{M}\right) \rightarrow\left(N^{3}, c_{N}, D^{N}\right)$ is a harmonic morphism.

(a2) $\varphi:\left(M^{4}, c_{M}\right) \rightarrow\left(N^{3}, c_{N}, D^{N}\right)$ is twistorial.

(a3) The fibres of $\Phi$ are tangent to $\mathscr{D}^{M}$.

(b) The following assertions are equivalent:

(b1) There exists a Weyl connection $D^{N}$ on $\left(N^{3}, c_{N}\right)$ and a section $k$ of the dual of the line bundle $L_{N}$ of $\left(N^{3}, c_{N}\right)$ such that $\varphi:\left(M^{4}, c_{M}, D^{M}\right) \rightarrow\left(N^{3}, c_{N}, D^{N}\right)$ is a twistorial harmonic morphism, and the vertical component of $D^{M}-D$ is equal to $\frac{1}{2} k$, under the isomorphism $\mathscr{V}=\varphi^{*}\left(L_{N}\right)$ corresponding to the orientation of $\left(M^{4}, c_{M}\right)$.

(b2) $\mathscr{D}^{M}$ is projectable with respect to $\Phi$ onto a three-dimensional distribution on $P_{N}$.

(b3) There exists a Weyl connection $D^{N}$ on $\left(N^{3}, c_{N}\right)$ and a section $k$ of $L_{N}^{*}$ such that $d \Phi\left(\mathscr{D}^{M}\right)=\mathscr{D}^{\nabla}$, where $\mathscr{D}^{\nabla} \subseteq T P_{N}$ is the connection on $P_{N}$ induced by the connection $\nabla$ on $L_{N}^{*} \otimes T N$ defined by

$$
\nabla_{X} \xi=D_{X}^{N} \xi+\frac{1}{2} k X \times \xi
$$

for any local sections $X$ of $T N$ and $\xi$ of $L_{N}^{*} \otimes T N$.

Furthermore, if assertions (b1), (b2) and (b3) hold, then $D^{N}$ and $k$ are determined by

$$
D^{M}=D+\frac{1}{2}\left(k+* \mathscr{H}^{I^{\mathscr{H}}}\right) \quad \text { and } \quad D^{N}=D_{+} .
$$

Proof. To prove (a), we claim that (a3) is equivalent to the following equality of partial connections, over $\mathscr{H}$,

$$
\mathscr{H} D^{M}=\mathscr{H} D+\frac{1}{2} * \mathscr{H} I^{\mathscr{H}} .
$$

Indeed, note that (a3) holds if and only if for any positively oriented conformal local frame $\left(X_{0}, X_{1}, X_{2}, X_{3}\right)$ such that $X_{0}$ is tangent to the fibres of 
$\varphi$ and $X_{1}, X_{2}, X_{3}$ are basic, we have

$$
g\left(D_{X_{0}}^{M}\left(X_{0}+i X_{1}\right), X_{2}+i X_{3}\right)=0
$$

where $g$ is the local representative of $c_{M}$ determined by $\left(X_{0}, X_{1}, X_{2}, X_{3}\right)$. An easy calculation shows that (5.4) is equivalent to

$$
\left(\alpha_{M}-\left(\operatorname{trace}_{g}\left(B^{\mathscr{V}}\right)^{b}+\frac{1}{2} * \mathscr{H} I^{\mathscr{H}}\right)\right)\left(X_{2}+i X_{3}\right)=0
$$

where $\alpha_{M}$ is the Lee form of $D^{M}$ with respect to $g$. Now, the equivalence (a3) $\Longleftrightarrow$ (5.3) follows easily.

Assertion (a) follows from Proposition 3.2 and Example 5.3.

To prove (b), first note that (b3) $\Longrightarrow(\mathrm{b} 2)$ is trivial. Conversely, if (b2) holds, then as $\pi_{N}$ is proper, $d \Phi\left(\mathscr{D}^{M}\right)$ is a connection on $P_{N}$ whose holonomy group is contained in the group of (complex analytic) diffeomorphisms of $\mathbb{C} P^{1}$. Hence, the holonomy group of $d \Phi\left(\mathscr{D}^{M}\right)$ is contained in $P G L(2, \mathbb{C})(=$ $\left.S L(2, \mathbb{C}) / \mathbb{Z}_{2}\right)$. As $S O(3, \mathbb{C})$ is the adjoint group of $S L(2, \mathbb{C})$, we have $S O(3, \mathbb{C})=S L(2, \mathbb{C}) / \mathbb{Z}_{2}$. Therefore, any connection on $P_{N}$ corresponds to a connection on the oriented Riemannian bundle $\left(L_{N}^{*} \otimes T N, c_{N}\right)$. In particular, there exists a unique connection $\nabla$ on $\left(L_{N}^{*} \otimes T N, c_{N}\right)$, which induces the connection $d \Phi\left(\mathscr{D}^{M}\right)$ on $P_{N}$. Note that $\nabla$ canonically determines a projective structure on $N^{3}$ which has the property that any of its geodesics which is null at some point is null everywhere. Furthermore, a null curve on $\left(N^{3}, c_{N}\right)$ is a geodesic of $\nabla$ if and only if its velocity vector field $Y$ has the property that $Y^{\perp}$ is parallel with respect to $\nabla$. From the fact that $\varphi$ is horizontally conformal, it follows that $\nabla$ and $c_{N}$ have the same null geodesics; equivalently, there exists a Weyl connection $D^{N}$ on $\left(N^{3}, c_{N}\right)$ and a section $k$ of $L_{N}^{*}$ so that (5.1) holds. Thus, we have proved that (b2) $\Longrightarrow(\mathrm{b} 3)$.

It is obvious that if (b3) holds, then $\nabla$ and $D^{N}$ determine the same projective structure. It follows that $\varphi:\left(M^{4}, c_{M}\right) \rightarrow\left(N^{3}, c_{N}, D^{N}\right)$ is twistorial. Thus, by (a), if (b3) holds, then $\varphi:\left(M^{4}, c_{M}, D^{M}\right) \rightarrow\left(N^{3}, c_{N}, D^{N}\right)$ is a twistorial harmonic morphism. Further, as $\varphi:\left(M^{4}, c_{M}\right) \rightarrow\left(N^{3}, c_{N}, D^{N}\right)$ is twistorial, the partial connection over $\mathscr{H}$ determined by the pull-back of $D^{N}$ by $\varphi$ is equal to $D_{+}$. Also, an argument similar to the proof of (a) shows that a field $p$ of self-dual spaces over a horizontal curve on $\left(M^{4}, c_{M}\right)$ is parallel with respect to $D^{M}$ if and only if $\mathscr{H} p$ is parallel with respect to the partial connection $\widehat{\nabla}$ on $\mathscr{V}^{*} \otimes \mathscr{H}$, over $\mathscr{H}$, defined by

$$
\widehat{\nabla}_{X} \xi=\left(D_{+}\right)_{X} \xi+\frac{1}{2} \widehat{k} X \times \xi
$$


for any local sections $X$ of $\mathscr{H}$ and $\xi$ of $\mathscr{V}^{*} \otimes \mathscr{H}$, where $\widehat{k}$ is the section of $\mathscr{V}^{*}$ defined by

$$
D^{M}=D+\frac{1}{2}\left(\widehat{k}+* \mathscr{H} I^{\mathscr{H}}\right) .
$$

Thus, the partial connection over $\mathscr{H}$ induced by the pull-back of $\nabla$ by $\varphi$ is equal to $\widehat{\nabla}$. Consequently, $\widehat{k}$ is the pull-back of $k$ by $\varphi$. In particular, (b3) $\Longrightarrow(\mathrm{b} 1)$.

We have proved that (b3) is equivalent to the existence of a Weyl connection $D^{N}$ on $\left(N^{3}, c_{N}\right)$ and a basic section $k$ of $\mathscr{V}^{*}\left(=\varphi^{*}\left(L_{N}^{*}\right)\right)$ such that (5.2) holds. On the other hand, by the proof of (a), the map $\varphi:\left(M^{4}, c_{M}, D^{M}\right) \rightarrow$ $\left(N^{3}, c_{N}, D^{N}\right)$ is a twistorial harmonic morphism for some Weyl connection $D^{N}$ on $\left(N^{3}, c_{N}\right)$ if and only if there exists a section $k$ of $\mathscr{V}^{*}$ such that (5.2) holds. Thus, (b1) $\Longrightarrow(\mathrm{b} 3)$.

The theorem is proved.

Remark 5.5. Let $\varphi:\left(M^{4}, c_{M}, D^{M}\right) \rightarrow\left(N^{3}, c_{N}, D^{N}\right)$ be a twistorial harmonic morphism with nowhere degenerate fibres. From Theorems 3.4 and 5.4(a), it follows that the fibres of $\varphi$ are geodesics with respect to $D^{M}$ if and only if $\mathscr{H}$ is integrable; in particular, $\varphi$ is also twistorial with respect to the reversed orientation of $\left(M^{4}, c_{M}\right)$, and hence, the fibres of $\varphi$ are locally generated by nowhere zero conformal vector fields.

For later use, we formulate the following definition (cf. $[7,10])$.

Definition 5.6. Let $\left(N^{3}, c, D\right)$ be a three-dimensional Weyl space. We say that $\left(N^{3}, c, D\right)$ is a Gauduchon-Tod space if there exist three onedimensional conformal foliations by geodesics $\mathscr{V}_{1}, \mathscr{V}_{2}, \mathscr{V}_{3}$ which are orthogonal on each other and such that $\mathscr{V}_{j}^{\perp}$, considered with the conformal structure induced by $c$, is orientable, $j=1,2,3$.

Remark 5.7. Let $\left(N^{3}, c\right)$ be a three-dimensional conformal manifold, and let $L$ be the associated line bundle. Then, it is known that, locally, the following assertions are equivalent (the equivalences (i) $\Longleftrightarrow$ (ii) $\Longleftrightarrow$ (iii) are proved in [10] while (i) $\Longleftrightarrow$ (iv) $\Longleftrightarrow$ (v) follow from [13]):

(i) There exists a Weyl connection $D$ on $\left(N^{3}, c\right)$ with respect to which $\left(N^{3}, c, D\right)$ is Gauduchon-Tod.

(ii) There exists an Einstein-Weyl connection $D$ on $\left(N^{3}, c\right)$ whose scalar curvature $s^{D}$ is given by $s^{D}=\frac{3}{2} k^{2}$ for a section $k$ of $L^{*}$ which satisfies $*_{c} D k=F^{D}$, where $F^{D}$ is the curvature form of the connection, on $L$, corresponding to $D$. 
(iii) There exists a Weyl connection $D$ on $\left(N^{3}, c\right)$ and a section $k$ of $L^{*}$ such that the connection $\nabla$ on $L^{*} \otimes T N$, defined by

$$
\nabla_{X} \xi=D_{X} \xi+\frac{1}{2} k X \times \xi
$$

for any local sections $X$ of $T N$ and $\xi$ of $L^{*} \otimes T N$, is flat.

(iv) There exists a flat connection on $\left(L^{*} \otimes T N, c\right)$ for which the associated twistor distribution on the bundle of two-dimensional degenerate spaces on $\left(N^{3}, c\right)$ is integrable.

(v) There exists an Einstein-Weyl connection $D$ on $\left(N^{3}, c\right)$ for which there exists a submersion from its twistor space onto $\mathbb{C} P^{1}$ whose fibres are transversal to the twistor lines.

We end this section with the following result.

Corollary 5.8. Let $\varphi:\left(M^{4}, c_{M}, D^{M}\right) \rightarrow\left(N^{3}, c_{N}, D^{N}\right)$ be a twistorial harmonic morphism with nowhere degenerate fibres from an oriented Weyl space of dimension 4 to a Weyl space of dimension 3 ; let $k \in \Gamma\left(\mathscr{V}^{*}\right)$ be the vertical component of $2\left(D^{M}-D\right)$. Then, locally, the following assertions are equivalent:

(i) $D^{M}$ is the Obata connection of a hyper-Hermitian structure on $\left(M^{4}, c_{M}\right)$.

(ii) $\left(N^{3}, c_{N}, D^{N}\right)$ is Gauduchon-Tod and $*_{N} D^{N} k=F^{D^{N}}$.

(iii) $\left(M^{4}, c_{M}, D^{M}\right)$ is Einstein-Weyl anti-self-dual and $k$ is basic.

Conversely, let $\varphi:\left(M^{4}, c_{M}\right) \rightarrow\left(N^{3}, c_{N}, D^{N}\right)$ be a twistorial map, with nowhere degenerate fibres, from an oriented conformal manifold of dimension 4 to a Gauduchon-Tod space, and let $D^{M}$ be the Obata connection of the hyper-Hermitian structure induced on $\left(M^{4}, c_{M}\right)$. Then, $\varphi:\left(M^{4}, c_{M}, D^{M}\right) \rightarrow$ $\left(N^{3}, c_{N}, D^{N}\right)$ is a harmonic morphism.

Proof. With the same notations as in Theorem 5.4, let $\mathscr{F} \subseteq \mathscr{D}^{M}$ be the twistor distribution defined by $\mathscr{F}_{p} \subseteq T_{p} P_{M}$ is the horizontal lift of $p \subseteq T_{\pi_{M}(p)} M$ with respect to $D^{M},\left(p \in P_{M}\right)$. Then, $\left(M^{4}, c_{M}\right)$ is anti-selfdual if and only if $\mathscr{F}$ is integrable. Also, $\left(M^{4}, c_{M}, D^{M}\right)$ is Einstein-Weyl anti-self-dual if and only if $\mathscr{D}^{M}$ is projectable with respect to $\mathscr{F}$ (that is, $[X, Y]$ is a local section of $\mathscr{D}^{M}$ if $X$ and $Y$ are local sections of $\mathscr{D}^{M}$ and $\mathscr{F}$, respectively). 
As (i) is satisfied if and only if the connection $\mathscr{D}^{M}$ is trivial, the equivalence (i) $\Longleftrightarrow$ (ii) follows from Theorem 5.4 and Remark 5.7.

If (i) holds, from Theorem 5.4(a) we obtain that $\mathscr{D}^{M}$ is projectable with respect to $\Phi$. Thus, by Theorem $5.4(\mathrm{~b}), k$ is basic. Hence, (i) $\Longrightarrow$ (iii).

If (iii) holds, then by Theorem $5.4(\mathrm{~b}), \mathscr{D}^{M}$ is projectable with respect to $\Phi$. It follows easily that $\mathscr{D}^{M}$ is integrable. Thus, locally, (iii) $\Longrightarrow(\mathrm{i})$.

The converse statement follows from Theorem 5.4(b).

\section{Relations between the twistoriality of harmonic morphisms and the Ricci tensor}

In this section, we shall work in the complex analytic category. Here, by a real conformal manifold, we mean the (germ-unique) complexification of a real analytic conformal manifold; similarly, we sometimes work with real Weyl spaces.

The following lemma follows from a straightforward computation.

Lemma 6.1 (cf. [2]). Let $(M, c, D)$ be a Weyl space, $\operatorname{dim} M=3,4$, and let Ric be its Ricci tensor. Let $\mathscr{F}$ be a foliation by null geodesics on $(M, c, D)$ such that $\mathscr{F}^{\perp}$ is integrable.

(i) If $\operatorname{dim} M=3$, then

$$
\operatorname{Ric}(Y, Y)=Y\left(g\left(D_{U} U, Y\right)\right)-g\left(D_{U} U, Y\right)^{2},
$$

where $\{U, Y, \tilde{Y}\}$ is a local frame on $M$ such that $Y$ is a local section of $\mathscr{F}, D_{Y} Y=0$ and $g=U \odot U+2 Y \odot \widetilde{Y}$ is a local representative of $c$.

(ii) If $\operatorname{dim} M=4$, then

$$
\operatorname{Ric}(Y, Y)=2\left[Y\left(g\left(D_{U} \widetilde{U}, Y\right)\right)-g\left(D_{U} \widetilde{U}, Y\right)^{2}-g([U, Y], U) g([\widetilde{U}, Y], \widetilde{U})\right]
$$

where $\{U, \widetilde{U}, Y, \widetilde{Y}\}$ is a local frame on $M$ such that $Y$ is a local section of $\mathscr{F}, D_{Y} Y=0$ and $g=2(U \odot \widetilde{U}+Y \odot \widetilde{Y})$ is a local representative of $c$.

\section{Remark 6.2.}

(1) In Lemma 6.1(i), the condition $\mathscr{F}^{\perp}$ integrable is superfluous. It follows that from any three-dimensional conformal manifold, we can, locally, define horizontally conformal submersions with one-dimensional nowhere degenerate fibres tangent to any given direction at a point. A 
similar statement holds for real analytic three-dimensional conformal manifolds.

(2) A relation slightly longer than in Lemma 6.1(ii) can be obtained for a foliation $\mathscr{F}$ by null geodesics on a four-dimensional Weyl space, without the assumption $\mathscr{F}^{\perp}$ is integrable.

Proposition 6.3 (cf. [2,29]). Let $\left(M, c_{M}, D^{M}\right)$ be a Weyl space, and let $\left(N, c_{N}\right)$ be a conformal manifold, $\operatorname{dim} M=3,4, \operatorname{dim} N=2$.

Let $\varphi:\left(M, c_{M}\right) \rightarrow\left(N, c_{N}\right)$ be a horizontally conformal submersion with nowhere degenerate fibres; if $\operatorname{dim} M=4$, assume $\varphi$ real (that is, $\varphi$ is the (germ-unique) complexification of a real analytic map and, in particular, $\left(M, c_{M}, D^{M}\right)$ and $\left(N, c_{N}\right)$ are complexifications of a real analytic Weyl space and a real analytic conformal manifold, respectively).

If trace $_{c_{M}}(D d \varphi)=0$ along a nondegenerate hypersurface foliated by the fibres of $\varphi$, then any two of the following assertions imply the third:

(i) $\varphi:\left(M, c_{M}, D^{M}\right) \rightarrow\left(N, c_{N}\right)$ is a harmonic morphism.

(ii) $\varphi:\left(M, c_{M}, D^{M}\right) \rightarrow\left(N, c_{N}\right)$ is twistorial.

(iii) The trace-free symmetric part of the horizontal component of the Ricci tensor of $D^{M}$ is zero.

Proof. If $\operatorname{dim} M=3$, then (i) $\Longleftrightarrow$ (ii). Also, we can find a local frame $\{U, Y, \widetilde{Y}\}$, as in Lemma 6.1(i), such that $U$ is tangent to the fibres of $\varphi$. Then, assertion (ii) is equivalent to $g\left(D_{U}^{M} U, Y\right)=g\left(D_{U}^{M} U, \widetilde{Y}\right)=0$. On the other hand, assertion (iii) is equivalent to ${ }^{M} \operatorname{Ric}(Y, Y)={ }^{M} \operatorname{Ric}(\widetilde{Y}, \widetilde{Y})=0$, where ${ }^{M}$ Ric is the Ricci tensor of $D^{M}$. Thus, if $\operatorname{dim} M=3$, the proof follows from Lemma 6.1(i).

Suppose $\operatorname{dim} M=4$. Then we can find a local frame $\{U, \widetilde{U}, Y, \widetilde{Y}\}$ like in Lemma 6.1(ii) such that $U$ and $\widetilde{U}$ are tangent to the fibres of $\varphi$. Moreover, we may assume $g$ oriented such that $\mathscr{F}_{+}=\operatorname{Span}(U, Y)$ and $\widetilde{\mathscr{F}}_{+}=\operatorname{Span}(\widetilde{U}, \widetilde{Y})$ are self-dual while $\mathscr{F}_{-}=\operatorname{Span}(\widetilde{U}, Y)$ and $\widetilde{\mathscr{F}}_{-}=\operatorname{Span}(U, \tilde{Y})$ are anti-selfdual. Then assertion (ii) is equivalent to the fact that either $\mathscr{F}_{+}, \widetilde{\mathscr{F}}_{+}$ are integrable or $\mathscr{F}_{-}, \widetilde{F}_{-}$are integrable. On the other hand, assertion (i) is equivalent to $g\left(D_{U}^{M} \widetilde{U}, Y\right)=g\left(D_{U}^{M} \widetilde{U}, \widetilde{Y}\right)=0$ (see the proof of Proposition 4.9). Thus, if $\operatorname{dim} M=4$, the proof follows from Lemma 6.1(ii).

\section{Remark 6.4.}

(1) If $\operatorname{dim} M=3$, then in the hypotheses of Proposition 6.3, assertions (i), (ii), (iii) are equivalent. 
It follows that if $\left(M, c_{M}, D^{M}\right)$ is a three-dimensional Weyl space from which there can be locally defined more than $k=6$ harmonic morphisms with one-dimensional nowhere degenerate fibres, then $\left(M, c_{M}\right.$, $\left.D^{M}\right)$ is Einstein-Weyl; in the smooth category, the same statement holds with $k=2$ ([7], cf. [2]).

(2) In the smooth category, suppose assertion (i) of Proposition 6.3 holds.

(a) If $\operatorname{dim} M=3$, then assertion (iii) also holds (cf. [2]).

(b) If $\operatorname{dim} M=4$, then the implication (ii) $\Rightarrow$ (iii) holds on $M$ while the implication (iii) $\Rightarrow$ (ii) holds locally on a dense open set of $M$ (cf. [29]).

(3) Proposition 6.3 also holds for any horizontally conformal submersion $\varphi:\left(M, c_{M}, D^{M}\right) \rightarrow\left(N, c_{N}, D^{N}\right)$ with nowhere degenerate fibres from a Weyl space to an Einstein-Weyl space, $\operatorname{dim} M=4, \operatorname{dim} N=3$ (see Proposition 6.6 below for an extension of this fact).

(4) For the implications (i), (ii) $\Rightarrow$ (iii) and (ii), (iii) $\Rightarrow$ (i) of Proposition 6.3, it is not necessary to assume that $\varphi$ is real when $\operatorname{dim} M=4$.

The proof of the following lemma is omitted.

Lemma 6.5 (cf. [25]). Let $\varphi:\left(M, c_{M}, D^{M}\right) \rightarrow\left(N, c_{N}, D^{N}\right)$ be a submersive harmonic morphism with nowhere degenerate fibres between Weyl spaces, $\operatorname{dim} M=4, \operatorname{dim} N=3$.

Let $A_{ \pm}=D_{ \pm}-D^{N}$. Then for any horizontal null vector $Y$, we have

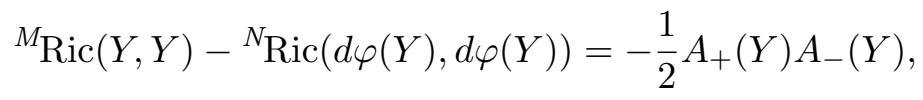

where ${ }^{M}$ Ric and ${ }^{N}$ Ric are the Ricci tensors of $D^{M}$ and $D^{N}$, respectively.

The following result follows from Lemmas 6.1(ii) and 6.5.

Proposition 6.6 (cf. [25]). Let $\varphi:\left(M, c_{M}, D^{M}\right) \rightarrow\left(N, c_{N}, D^{N}\right)$ be a nonconstant harmonic morphism with nowhere degenerate fibres between Weyl spaces, $\operatorname{dim} M=4, \operatorname{dim} N=2,3$. If $\operatorname{dim} N=2$, suppose that $\varphi$ is real.

Let ${ }^{M}$ Ric and ${ }^{N}$ Ric be the Ricci tensors of $D^{M}$ and $D^{N}$, respectively. Then the following assertions are equivalent:

(i) $\varphi$ is twistorial.

(ii) The trace-free symmetric part of the horizontal component of ${ }^{M} \mathrm{Ric}-\varphi^{*}\left({ }^{N} \mathrm{Ric}\right)$ is zero. 


\section{Harmonic morphisms from four-dimensional Einstein-Weyl spaces}

In this section, we shall work in the complex analytic category; here, as in the previous section, by a real conformal manifold (Weyl space), we mean the complexification of a real analytic conformal manifold (Weyl space).

From Proposition 6.6, we obtain the following two corollaries.

Corollary 7.1 (cf. [26, 29]). Let $\varphi:\left(M^{4}, c_{M}, D^{M}\right) \rightarrow\left(N^{2}, c_{N}\right)$ be a real submersive harmonic morphism from a four-dimensional real Einstein-Weyl space to a two-dimensional real conformal manifold.

Then, $\varphi:\left(M^{4}, c_{M}\right) \rightarrow\left(N^{2}, c_{N}\right)$ is a twistorial map. Furthermore, if $\left(M^{4}, c_{M}\right)$ is nonorientable, then the fibres of $\varphi$ are totally geodesic.

Corollary 7.2 (cf. $[23,25,26])$. Let $\left(M^{4}, c_{M}, D^{M}\right)$ be an Einstein-Weyl space of dimension 4 , and let $\varphi:\left(M^{4}, c_{M}, D^{M}\right) \rightarrow\left(N^{3}, c_{N}, D^{N}\right)$ be a submersive harmonic morphism with nowhere degenerate fibres to a Weyl space of dimension 3.

Then, $\varphi:\left(M^{4}, c_{M}\right) \rightarrow\left(N^{3}, c_{N}, D^{N}\right)$ is a twistorial map if and only if $D^{N}$ is an Einstein-Weyl connection.

Remark 7.3. We do not know whether or not it is true that if $\left(M^{4}, c_{M}, D^{M}\right)$ is Einstein-Weyl and $\varphi:\left(M^{4}, c_{M}, D^{M}\right) \rightarrow\left(N^{3}, c_{N}, D^{N}\right)$ is a submersive harmonic morphism with nowhere degenerate fibres, then, locally, there exists a Weyl connection $D$ on $\left(N^{3}, c_{N}\right)$ with respect to which $\varphi:\left(M^{4}, c_{M}\right) \rightarrow\left(N^{3}, c_{N}, D\right)$ is twistorial.

From $[23,26]$, it follows that this holds if $D^{M}$ and $D^{N}$ are Levi-Civita connections of representatives of $c_{M}$ and $c_{N}$, respectively. Furthermore, if we also assume $D \neq D^{N}$, then $\varphi$ must be of Killing type; for example, the Killing vector field $V=x_{1} \partial / \partial x_{2}-x_{2} \partial / \partial x_{1}$ on $\mathbb{C}^{4}$ defines such harmonic morphisms (this follows from Example 5.3 by noting that $V^{\perp}$ is integrable but the nondegenerate orbits of $V$ are nowhere geodesic).

Next, we give the necessary and sufficient conditions under which on a real Einstein-Weyl space of dimension 4 there can be defined, locally, at least five distinct real foliations of dimension 2 which produce harmonic morphisms (cf. Remark 6.4(1)).

Theorem 7.4. Let $\left(M^{4}, c_{M}, D^{M}\right)$ be an orientable real Einstein-Weyl space of dimension 4. 
Then, locally, there can be defined on $\left(M^{4}, c_{M}, D^{M}\right)$ five distinct real foliations of dimension 2 which produce harmonic morphisms if and only if one of the following two assertions holds:

(i) $D^{M}$ is the Weyl connection of a Hermitian structure locally defined on $\left(M^{4}, c_{M}\right)$;

(ii) $\left(M^{4}, c_{M}\right)$ is anti-self-dual with respect to a suitable orientation, and $D^{M}$ is the Levi-Civita connection of a local Einstein representative of $c_{M}$.

Proof. Let $\mathscr{V}_{k}, k=1, \ldots, 5$, be five distinct two-dimensional foliations which produce harmonic morphisms on $\left(M^{4}, c_{M}, D^{M}\right)$. By passing to a conformal covering, if necessary, we may assume that $\mathscr{V}_{k}, k=1, \ldots, 5$, endowed with the conformal structures induced by $c_{M}$, are orientable. Also, as $M^{4}$ is locally compact, we may suppose that $\mathscr{V}_{k}(x), k=1, \ldots, 5$, are distinct at each $x \in M$.

By Corollary 7.1 , for each $k=1, \ldots, 5$, we have $\mathscr{V}_{k}$ twistorial with respect to a suitable orientation on $\left(M^{4}, c_{M}\right)$. Thus, there exists an orientation on $\left(M^{4}, c_{M}\right)$ with respect to which, after a renumbering, $\mathscr{V}_{k}$ is twistorial for $k=1,2,3$.

Let $J_{k}$ be the positive Hermitian structures on $\left(M^{4}, c_{M}\right)$, uniquely determined up to sign, such that $J_{k}\left(\mathscr{V}_{k}\right)=\mathscr{V}_{k}, k=1,2,3$. Then, either there exists $k \in\{1,2,3\}$ such that $D^{M}$ is the Weyl connection of $\left(M^{4}, c_{M}, J_{k}\right)$ or for any $k \in\{1,2,3\}$, we have $D^{M} J_{k} \neq 0$. In the latter case, from Proposition 4.9 , it follows that for $k=1,2,3$, the complex structure $J_{k}$ determines $\mathscr{V}_{k}[29]$, and hence we obtain that $J_{k} \pm J_{l} \neq 0$ for $k \neq l$; thus, $\left(M^{4}, c_{M}\right)$ is anti-self-dual (see [27]). Now, as $\left(M^{4}, c_{M}, D^{M}\right)$ is anti-self-dual and Einstein-Weyl, by a result of Pedersen, Swann and Calderbank (see [6] and the references therein), we have that, locally, either $D^{M}$ is the Obata connection of a hyper-Hermitian structure on $\left(M^{4}, c_{M}\right)$ or $D^{M}$ is the Levi-Civita connection of an Einstein representative of $c_{M}$.

Conversely, if (i) or (ii) holds, then, locally, there can be defined infinitely many two-dimensional foliations on $\left(M^{4}, c_{M}, D^{M}\right)$ which produce harmonic morphisms. Indeed if $\left(M^{4}, c_{M}, D^{M}\right)$ satisfies assertion (i), then this follows easily from Remark 4.3(ii), while if $\left(M^{4}, c_{M}, D^{M}\right)$ satisfies assertion (ii), then this is, essentially, proved in [29]. The theorem is proved.

Remark 7.5. Let $\left(M^{4}, c_{M}, D^{M}\right)$ be an orientable four-dimensional Einstein-Weyl space endowed either with nine distinct foliations by degenerate hypersurfaces, which produce harmonic morphisms, or with thirteen 
distinct foliations, by nondegenerate surfaces, which produce harmonic morphisms.

Then, arguments as in the proof of Theorem 7.4 show that up to conformal covering spaces, one of the following two assertions holds:

(i) The connection induced by $D^{M}$ on the bundle of self-dual spaces on $\left(M^{4}, c_{M}\right)$, considered with a suitable orientation, admits a reduction to the group of affine transformations of $\mathbb{C}$.

(ii) $\left(M^{4}, c_{M}\right)$ is anti-self-dual with respect to a suitable orientation, and $D^{M}$ is the Levi-Civita connection of an Einstein representative of $c_{M}$.

Finally, we describe the harmonic morphisms with nowhere degenerate fibres between Einstein-Weyl spaces of dimensions 4 and 3 .

Theorem 7.6. Let $\left(M^{4}, c_{M}, D^{M}\right)$ and $\left(N^{3}, c_{N}, D^{N}\right)$ be Einstein-Weyl spaces of dimensions 4 and 3, respectively, and let $\varphi: M^{4} \rightarrow N^{3}$ be a submersion with nowhere degenerate fibres.

If $\left(M^{4}, c_{M}\right)$ is orientable, then $\varphi:\left(M^{4}, c_{M}, D^{M}\right) \rightarrow\left(N^{3}, c_{N}, D^{N}\right)$ is a harmonic morphism if and only if $\left(M^{4}, c_{M}\right)$ is anti-self-dual with respect to a suitable orientation, $\varphi:\left(M^{4}, c_{M}\right) \rightarrow\left(N^{3}, c_{N}, D^{N}\right)$ is twistorial and, locally, either

(i) $\left(N^{3}, c_{N}, D^{N}\right)$ is Gauduchon-Tod and $D^{M}$ is the Obata connection of the hyper-Hermitian structure induced on $\left(M^{4}, c_{M}\right)$, or

(ii) $D^{M}$ is the Levi-Civita connection of an Einstein representative $g$ of $c_{M}$ with nonzero scalar curvature, and the fibres of the twistorial representation $Z(\varphi): Z(M) \rightarrow Z(N)$ of $\varphi$ are tangent to the contact distribution on $Z(M)$ corresponding to $g$.

If $\left(M^{4}, c_{M}\right)$ is nonorientable, then $\varphi:\left(M^{4}, c_{M}, D^{M}\right) \rightarrow\left(N^{3}, c_{N}, D^{N}\right)$ is a harmonic morphism if and only if, locally, $D^{M}$ and $D^{N}$ are the Levi-Civita connections of constant curvature representatives of $c_{M}$ and $c_{N}$, respectively, and $\varphi$ is a harmonic morphism of warped product type.

Proof. If $\varphi:\left(M^{4}, c_{M}\right) \rightarrow\left(N^{3}, c_{N}, D^{N}\right)$ is twistorial and (i) or (ii) holds, then (a2) and (a3) of Theorem 5.4 are satisfied and hence $\varphi:\left(M^{4}, c_{M}, D^{M}\right) \rightarrow$ $\left(N^{3}, c_{N}, D^{N}\right)$ is a harmonic morphism.

Conversely, if $\varphi:\left(M^{4}, c_{M}, D^{M}\right) \rightarrow\left(N^{3}, c_{N}, D^{N}\right)$ is a harmonic morphism, then, by Corollary 7.2 , we have that $\varphi:\left(M^{4}, c_{M}\right) \rightarrow\left(N^{3}, c_{N}, D^{N}\right)$ is a 
twistorial map. From ([5], see [26]), it follows that either $\left(M^{4}, c_{M}\right)$ is orientable and anti-self-dual, with respect to a suitable orientation, or $\left(M^{4}, c_{M}\right)$ is flat.

If $\left(M^{4}, c_{M}\right)$ is orientable, as $\left(M^{4}, c_{M}, D^{M}\right)$ is Einstein-Weyl, then, as in the proof of Theorem 7.4, we have that, locally, either $D^{M}$ is the Obata connection of a hyper-Hermitian structure on $\left(M^{4}, c_{M}\right)$ or $D^{M}$ is the LeviCivita connection of an Einstein representative of $c_{M}$; if $\left(M^{4}, c_{M}\right)$ is nonorientable, then it must be flat, and hence, by a result of Eastwood and Tod, we have that, locally, $D^{M}$ is the Levi-Civita connection of some constant curvature representative of $c_{M}$ (see [6] and the references therein, and note that similar calculations prove that these two results also hold in the complex analytic category).

If $\left(M^{4}, c_{M}\right)$ is orientable, then from Theorem 5.4(a), it follows that, locally, we have the alternative (i) or (ii).

If $\left(M^{4}, c_{M}\right)$ is nonorientable, then by passing to its oriented $\mathbb{Z}_{2}$-covering, we obtain that $\varphi$ has integrable horizontal distribution. Hence, by Remark 5.5 , the fibres of $\varphi$ are geodesics of $D^{M}$. Furthermore, as $\left(N^{3}, c_{N}\right)$ can be, locally, identified with any leaf of the horizontal distribution of $\varphi$ endowed with the conformal structure induced by $c_{M}$, we have that $\left(N^{3}, c_{N}\right)$ is flat. Hence, locally, $D^{N}$ is the Levi-Civita connection of some constant curvature representative of $c_{N}$. Thus, if $\left(M^{4}, c_{M}\right)$ is nonorientable, then up to conformal coverings, $\varphi$ is a harmonic morphism with geodesic fibres and integrable horizontal distribution between Riemannian manifolds of constant curvature.

\section{Example 7.7.}

(1) The harmonic morphisms given by the Gibbons-Hawking and the Beltrami fields constructions (see [25]) satisfy assertion (i) of Theorem 7.6.

(2) The harmonic morphisms of warped product type with one-dimensional fibres from an oriented four-dimensional Riemannian manifold with nonzero constant sectional curvature satisfy assertion (ii) of Theorem 7.6. More generally, let $\left(M^{4}, g\right)$ be the $\mathcal{H}$-space [17] of the threedimensional conformal manifold $\left(N^{3}, c_{N}\right)$, and let $\nabla^{g}$ be the LeviCivita connection of $g$. Suppose that $\left(N^{3}, c_{N}\right)$ is endowed with an Einstein-Weyl connection $D^{N}$, and let $\varphi: M^{4} \rightarrow N^{3}$ be the (local) retraction [13] of $N^{3} \hookrightarrow M^{4}$ corresponding to $D^{N}$. Then, the map $\varphi:\left(M^{4},[g], \nabla^{g}\right) \rightarrow\left(N^{3}, c_{N}, D^{N}\right)$ is a harmonic morphism which satisfies assertion (ii) of Theorem 7.6. 


\section{Acknowledgments}

R.P. gratefully acknowledges that this work was partially supported by a grant from the Conseil Général du Finistère.

\section{References}

[1] P. Baird and J. Eells, A conservation law for harmonic maps, Geometry symposium, Utrecht 1980, Lect. Notes Math., 894, Springer-Verlag, Berlin, 1981, 1-25.

[2] P. Baird and J. C. Wood, Harmonic morphisms, Seifert fibre spaces and conformal foliations, Proc. London Math. Soc. 64 (1992) 170-196.

[3] P. Baird and J. C. Wood, Harmonic morphisms between Riemannian manifolds, London Mathematical Society Monographs (NS), 29, Oxford University Press, Oxford, 2003.

[4] R. Bott, Lectures on characteristic classes and foliations. Notes by Lawrence Conlon, with two appendices by J. Stasheff, Lectures on algebraic and differential topology (Second Latin American School in Math., Mexico City, 1971), Lecture Notes Math., 279, Springer, Berlin, 1972, $1-94$.

[5] D. M. J. Calderbank, Selfdual Einstein metrics and conformal submersions. Preprint, Edinburgh University, 2000 (available from http://www-users.york.ac.uk/ dc511/mpapers.html, math.DG/0001041).

[6] D. M. J. Calderbank, The Faraday 2-form in Einstein-Weyl geometry, Math. Scand. 89 (2001), 97-116.

[7] D. M. J. Calderbank and H. Pedersen, Selfdual spaces with complex structures, Einstein-Weyl geometry and geodesics, Ann. Inst. Fourier (Grenoble) 50 (2000), 921-963.

[8] B. Fuglede, Harmonic morphisms between Riemannian manifolds, Ann. Inst. Fourier (Grenoble) 28 (1978), 107-144.

[9] P. Gauduchon, Structures de Weyl-Einstein, espaces de twisteurs et variétés de type $\mathbf{S}^{1} \times \mathbf{S}^{3}$, J. Reine Angew. Math. 469 (1995), 1-50.

[10] P. Gauduchon and K. P. Tod, Hyper-Hermitian metrics with symmetry, J. Geom. Phys. 25 (1998), 291-304. 
[11] S. Gudmundsson, The bibliography of harmonic morphisms, http://www.maths.lth.se/matematiklu/personal/sigma/harmonic /bibliography.html

[12] S. Gudmundsson and J. C. Wood, Harmonic morphisms between almost Hermitian manifolds, Boll. Unione Mat. Ital. (7) 11B (Supplement to fasc. 1) (1997), 30-58.

[13] N. J. Hitchin, Complex manifolds and Einstein's equations, Twistor geometry and nonlinear systems (Primorsko, 1980), Lecture Notes Math., 970, Springer, Berlin, 1982, 73-99.

[14] T. Ishihara, A mapping of Riemannian manifolds which preserves harmonic functions, J. Math. Kyoto Univ. 19 (1979), 215-229.

[15] P. E. Jones and K. P. Tod, Minitwistor spaces and Einstein-Weyl spaces, Classical Quant. Grav. 2 (1985), 565-577.

[16] S. Kobayashi and K. Nomizu, Foundations of differential geometry, I, II, Wiley Classics Library (reprint of the 1963, 1969 original), WileyInterscience Publishers, Wiley, New-York, 1996.

[17] C. R. LeBrun, $\mathcal{H}$-space with a cosmological constant, Proc. Roy. Soc. London Ser. A 380 (1982), 171-185.

[18] A. Lichnerowicz, Applications harmoniques et variétés kähleriennes, Symposia Mathematica III (INDAM, Rome, 1968/1969), 341-402.

[19] E. Loubeau, Hermitian harmonic maps, Beiträge Algeb. Geom. 40 (1999), 1-14.

[20] E. Loubeau and R. Pantilie, Harmonic morphisms between Weyl spaces and twistorial maps II. Preprint, IMAR, 2006, (math.DG/0610676).

[21] L. J. Mason and N. M. J. Woodhouse, Integrability, self-duality, and twistor theory, London Mathematical Society Monographs (NS), 15, Oxford University Press, Oxford, 1996.

[22] A. Pambira, Harmonic morphisms between degenerate semiRiemannian manifolds, Beiträge Algeb. Geom. 46 (2005), 261-281.

[23] R. Pantilie, Harmonic morphisms with 1-dimensional fibres on 4-dimensional Einstein manifolds, Comm. Anal. Geom. 10 (2002), 779-814.

[24] R. Pantilie and J. C. Wood, Harmonic morphisms with one-dimensional fibres on Einstein manifolds, Trans. Amer. Math. Soc. 354 (2002), 4229-4243. 
[25] R. Pantilie and J. C. Wood, A new construction of Einstein self-dual manifolds, Asian J. Math. 6 (2002), 337-348.

[26] R. Pantilie and J. C. Wood, Twistorial harmonic morphisms with onedimensional fibres on self-dual four-manifolds, Q. J. Math. 57 (2006), $105-132$.

[27] S. Salamon, Special structures on four-manifolds. Conference on Differential Geometry and Topology (Italian) (Parma, 1991), Riv. Mat. Univ. Parma (4) 17* (1991), 109-123.

[28] I. Vaisman, On locally conformal almost Kähler manifolds, Israel J. Math. 24 (1976), 338-351.

[29] J. C. Wood, Harmonic morphisms and Hermitian structures on Einstein 4-manifolds, Internat. J. Math. 3 (1992), 415-439.

DÉPARTEMENT DE MATHÉMATiques

LABORATOIRE C.N.R.S. U.M.R. 6205

Université de Bretagne OCCIDENTAle

6, Avenue Victor Le Gorgeu

CS 93837

29238 BRest Cedex 3

FRANCE

E-mail address: Eric.Loubeau@univ-brest.fr

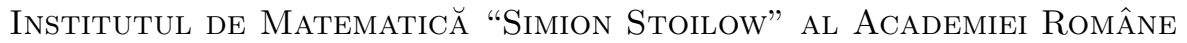
C.P. $1-764$

014700

BUCUREŞTI

ROMÂNIA

E-mail address: Radu.Pantilie@imar.ro

Received May 16, 2005 
